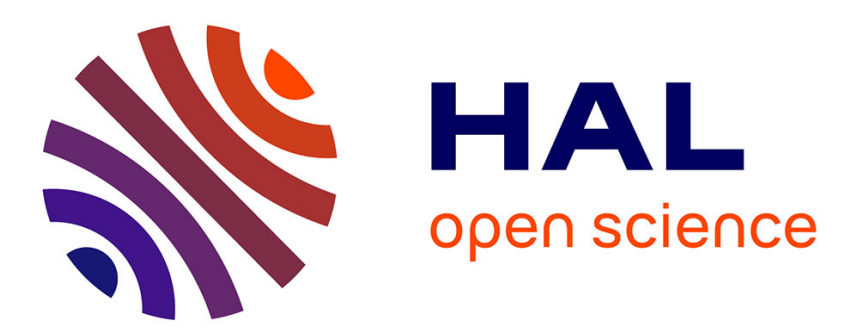

\title{
From the onset of damage to rupture: construction of responses with damage localization for a general class of gradient damage models
}

Kim Pham, Jean-Jacques Marigo

\section{- To cite this version:}

Kim Pham, Jean-Jacques Marigo. From the onset of damage to rupture: construction of responses with damage localization for a general class of gradient damage models. Continuum Mechanics and Thermodynamics, 2013, 25 (2-4), pp.147-171. 10.1007/s00161-011-0228-3 . hal-00647860

\section{HAL Id: hal-00647860 \\ https://hal.science/hal-00647860}

Submitted on 2 Dec 2011

HAL is a multi-disciplinary open access archive for the deposit and dissemination of scientific research documents, whether they are published or not. The documents may come from teaching and research institutions in France or abroad, or from public or private research centers.
L'archive ouverte pluridisciplinaire HAL, est destinée au dépôt et à la diffusion de documents scientifiques de niveau recherche, publiés ou non, émanant des établissements d'enseignement et de recherche français ou étrangers, des laboratoires publics ou privés. 


\title{
From the onset of damage to rupture: construction of responses with damage localization for a general class of gradient damage models
}

\author{
Kim Pham • Jean-Jacques Marigo
}

November 21, 2011

\begin{abstract}
We propose a construction method of non homogeneous solutions for the traction problem of an elastic damaging bar. This bar has a softening behavior which obeys a gradient damaged model. The method is applicable for a wide range of brittle materials. For sufficiently long bars, we show that localization arises on sets whose length is proportional to the material internal length and with a profile which is also a material characteristic. From its onset until the rupture, the damage profile is obtained either in a closed form or after a simple numerical integration depending on the model. Thus, the proposed method provides definitions for the critical stress and fracture energy that can be compared with experimental results. We finally discuss some features of the global behavior of the bar such as the possibility of a snap-back at the onset of damage. We point out the sensitivity of the responses to the parameters of the damage law. All these theoretical considerations are illustrated by numerical examples.
\end{abstract}

Keywords damage; gradient theory; localization; variational approach

PACS 02.30.Wd, 62.20.Mk, 68.35.Md, 81.40.Jj, 83.50.Lh

\section{Introduction}

It is possible to explain the rupture of materials with damage models. This can be done by means of the localization of damage on zones of small thickness where strain is large and stress is small. The choice of the type of damage model is essential to obtain valuable results. Rate independent local damage models where the local damage rate only depends on the local strain and damage state of the material are viable for hardening behavior. Nonetheless, they cease to give meaningful responses for softening behavior. Indeed, in this latter case the boundary-value problem is mathematically ill-posed (Benallal et al. (1993); Lasry and Belytschko (1988); Comi (1995)) in the sense that it admits an infinite number of linearly independent solutions. In particular, damage can concentrate on arbitrarily small zones and thus failure arises in the material without energy dissipation. Furthermore, numerical simulation with local models using the Finite Element Method are strongly mesh sensitive (Bažant et al. (1984); de Borst et al. (1993); Lasry and Belytschko (1988)). Two main regularization techniques exist to avoid these pathological localizations, namely the integral (Pijaudier-Cabot and Bažant (1987); Bažant and Pijaudier-Cabot (1988); Pijaudier-Cabot and Benallal (1993)) and the gradient (Peerlings et al. (1996, 1998); Comi (2001); Comi and Perego (2001); Lorentz et al. (2011)) damage approaches, see also Lorentz and Andrieux (2003) for an overview. Both consist in introducing non local terms in the model which contains accordingly (at least) one characteristic length.

In the present paper, we use gradient models in which the energy density function depends on the strain, the damage variable and the gradient of damage variable. One of the basic ingredients of our approach which differs from almost all previous works on the same subject consists in deriving the damage evolution problem from a

Kim Pham

Department of Mechanical Engineering, Eindhoven University of Technology, P.O. Box 513, 5600 MB Eindhoven, The Netherlands

E-mail: phammgoc.kimhung@gmail.com

Jean-Jacques Marigo

Laboratoire de Mécanique des Solides, Ecole Polytechnique, F91128 Palaiseau Cedex, France

E-mail: marigo@lms.polytechnique.fr 
variational approach based on an energetic formulation. Such energetic formulations - first introduced by Nguyen $(1987,2000)$ and then justified by Marigo $(1989,2000)$ (see also DeSimone et al. (2001)) by thermodynamical arguments for a large class of rate independent behaviors - constitute a very promising way to treat in a unified framework the questions of bifurcation and stability of solutions to quasi-static evolution problems. After the pioneering papers by Francfort and Marigo (1993, 1998), Mielke (2005) formalized these concepts for the very broad class of rate independent evolution laws, Bourdin et al. (2008) developed this approach in Fracture Mechanics, and Pham and Marigo (2010a,b) in Damage Mechanics.

The present paper is the natural continuation of Pham et al. (2011b) where the authors, first, studied the stability of the homogeneous response of a bar under an increasing traction loading at one end, and then the possibility of bifurcation from this homogeneous response ${ }^{1}$. The main result stated that a homogeneous state is stable provided the bar is short enough, the critical length being a material property which involves the internal length. Moreover this loss of stability is generally preceded by the possibility of bifurcation to a non homogeneous response. For sufficiently long bars, the loss of stability and the possibility of bifurcations occur at the end of the elastic phase while no damage has still appeared. But the construction of the bifurcated branches was not investigated in Pham et al. (2011b). Such a task is achieved in Benallal and Marigo (2007) but for a very particular gradient damage model. The goal of the present paper is to extend the results of Benallal and Marigo (2007) and to complete those of Pham et al. (2011a) for a large class of elastic-softening material. However, we will only focus on the construction of bifurcated branches corresponding to non-homogeneous solutions and the questions of stability will not be investigated here.

More specifically, the paper is structured as follows. In Section 2, we first define the general class of gradient damage models that are considered throughout the paper. These models where the damage variable is scalar are characterized by two state functions of the damage variable and one internal length. Then we set the damage evolution problem and deduce the local set of equations from its variational formulation. Section 3 is devoted to a step-wise construction of the non-homogeneous solutions where damage grows only in the so-called localization zones while the remaining part of the bar remains undamaged. Many properties are established and illustrated on several examples. In particular, the irreversibility of damage is one of the most difficult condition to ensure and is very discriminating in terms of the damage models. When it is satisfied, the construction can be performed up to the rupture. We finally obtain the damage profile and the involved fracture energy when the bar breaks, in terms of the parameters of the model. The last section is devoted to the analysis of the global behavior of the bar, in particular to the force-displacement responses. We discuss the number of possible solutions and the shape of the curves according to the bar length and the number of localization zones which grow. In particular, the possible presence of snap-backs and of discontinuous evolutions is discussed.

The following notation is used: the prime denotes either the spatial derivative or the derivative with respect to the damage parameter, the dot the time derivative, e.g. $u^{\prime}=\partial u / \partial x, E^{\prime}(\alpha)=d E(\alpha) / d \alpha, \dot{\alpha}=\partial \alpha / \partial t$. The qualifier increasing (resp. decreasing) stands for strictly increasing (resp. strictly decreasing) and should not be confused with non decreasing (resp. non increasing). In the same way, the qualifier positive (resp. negative) stands for $>0$ $($ resp. $<0)$ and not for $\geq 0$ (resp. $\leq 0)$.

\section{Setting of the damage problem}

\subsection{The gradient damage model}

We consider a one-dimensional gradient damage model in which the damage variable $\alpha$ is a real number growing from 0 to 1 , where $\alpha=0$ is the undamaged state and $\alpha=1$ is the full damaged state. The behavior of the material is characterized by the state function $W$ which gives the energy density at each point $x$. It depends on the local strain $\varepsilon(x)$ (if $u$ denotes the displacement field, then $\varepsilon(x)=u^{\prime}(x)$ where the prime stands for the spatial derivative), the local damage value $\alpha(x)$ and the local gradient $\alpha^{\prime}(x)$ of the damage field at $x$. Specifically, we assume that $W$ takes the following form

$$
W\left(\varepsilon, \alpha, \alpha^{\prime}\right)=\frac{1}{2} E(\alpha) \varepsilon^{2}+\mathrm{w}(\alpha)+\frac{1}{2} E_{0} \ell^{2} \alpha^{\prime 2} .
$$

In (1), $E_{0}$ represents the Young modulus of the undamaged material and $E(\alpha)$ the Young modulus of the material in the damage state $\alpha$. The second term $\mathrm{w}(\alpha)$ can be interpreted as the density of energy dissipated by the material during a homogeneous damage process (i.e. a process such that $\alpha^{\prime}(x)=0$ ) during which the damage variable grows from 0 to $\alpha$. The last term in (1) is the "non local" part of the energy which plays, as we will see in the next

\footnotetext{
1 Homogeneous response means that both the strain and the damage fields are the same at all points of the bar at a given time.
} 
section, a regularizing role by limiting the possibilities of localization of the damage field. For obvious reasons of physical dimension, this term involves a material characteristic length $\ell$ which will give the size of the damage localization zone. Denoting by $\sigma$ the stress, the stress-strain relation reads as

$$
\sigma=E(\alpha) \varepsilon
$$

The expression of the energy density (1) is analog to the one proposed by Comi and Perego (2001). It implicitly assumes a symmetric behavior in tension and compression. It must be modified to take into account asymmetric behaviors, like in Comi (2001) or Pham and Marigo (2010a,b). While remaining within the framework of symmetric behavior, a model which can seem more general would consist in replacing the constant $E_{0} \ell^{2}$ by a function of $\alpha$. It can be shown in fact that after an adequate change of variable, the damage parameter $\alpha$ can always be chosen so that the function becomes a constant, see Pham and Marigo (2010b). We thus assume here that the damage parameter has been chosen to this end. Note that another choice was made in Pham et al. (2011b).

The qualitative properties of the (gradient or local) model, in particular its softening or hardening character, strongly depend on some properties of the stiffness function $\alpha \mapsto E(\alpha)$, the dissipation function $\alpha \mapsto \mathrm{w}(\alpha)$, the compliance function $\alpha \mapsto S(\alpha)=1 / E(\alpha)$ and their derivatives. From now on we will adopt the following hypothesis:

Hypothesis 1 (Strongly brittle materials) $\alpha \mapsto E(\alpha)$ and $\alpha \mapsto \mathrm{w}(\alpha)$ are non negative and (at least) twice continuously differentiable functions on $[0,1)$ such that

$$
\begin{aligned}
& E(0)=E_{0}>0, \mathrm{w}(0)=0, \quad E^{\prime}(\alpha)<0, \mathrm{w}^{\prime}(\alpha)>0 \quad \forall \alpha \in[0,1), \quad E(1)=0, \mathrm{w}(1)<+\infty, \\
& -\mathrm{w}^{\prime}(\alpha) / E^{\prime}(\alpha) \text { is non decreasing and } \mathrm{w}^{\prime}(\alpha) / S^{\prime}(\alpha) \text { is decreasing to } 0 \text { when } \alpha \text { grows from } 0 \text { to } 1 .
\end{aligned}
$$

This corresponds to the family of strongly brittle materials with softening defined in Pham et al. (2011b).

Let us comment this Hypothesis before giving an example

1. The interval of definition of $\alpha$ can always be taken as $[0,1]$ after a change of the damage variable;

2. The condition $E^{\prime}<0$ denotes the decrease of the material stiffness when the damage grows;

3. The condition $E(1)=0$ ensures the total loss of stiffness when $\alpha=1$;

4. The positivity and the monotonicity of $w$ is natural since $w(\alpha)$ represents the energy dissipated during a damage process where the damage grows homogeneously in space from 0 to $\alpha$;

5. The boundedness of $w$ is characteristic of strongly brittle materials with softening; this condition disappears in the case of weakly brittle materials with softening or in the case of brittle materials with hardening;

6. The condition of monotonicity of $\mathrm{w}^{\prime} / E^{\prime}$ is introduced for the sake of simplicity and is unessential. In a homogeneous strain and damage response, it denotes that the strain does not decrease when the damage grows, see Section 2.3. This refers to the strain-hardening property;

7. The condition of monotonicity of $\mathrm{w}^{\prime} / S^{\prime}$ is essential; it denotes the softening property. In a homogeneous strain and damage response, this property leads to the decreasing of the corresponding stress when the damage grows, see Section 2.3;

8. The condition $\lim _{\alpha \rightarrow 1} \mathrm{w}^{\prime}(\alpha) / S^{\prime}(\alpha)=0$ ensures that the material cannot sustain any stress when its damage state is 1 .

Example 1 A family of models which satisfy the assumptions above is the following one, when $q>p>0$ :

$$
E(\alpha)=E_{0}(1-\alpha)^{q}, \quad \mathrm{w}(\alpha)=\frac{q \sigma_{c}^{2}}{2 p E_{0}}\left(1-(1-\alpha)^{p}\right)
$$

It contains five material parameters: the sound Young modulus $E_{0}>0$, the dimensionless parameters $p$ and $q$, the critical stress $\sigma_{c}>0$ and the internal length $\ell>0$ whose physical interpretation will be given in Section 2.3.

The condition $q>0$ is necessary and sufficient in order that $\alpha \mapsto E(\alpha)$ be decreasing from $E_{0}$ to 0 while the condition $p>0$ is necessary and sufficient in order that $\alpha \mapsto \mathrm{w}(\alpha)$ be increasing from 0 to a finite value. If $p>0$ and $q>0$, then the condition $q>p$ is necessary and sufficient in order that $\alpha \mapsto-\mathrm{w}^{\prime}(\alpha) / E^{\prime}(\alpha)$ be increasing to $\infty$ while $\alpha \mapsto \mathrm{w}^{\prime}(\alpha) / S^{\prime}(\alpha)$ is automatically decreasing to 0 .

A particularly interesting class of materials which satisfy the conditions of Hypothesis 1 is that of perfectly brittle materials which corresponds to the models in which $p=q>0$ in the previous example. By definition, these materials are such that $\mathrm{w}^{\prime} / E^{\prime}$ is constant. This leads to the following definition: 
Hypothesis 2 (Perfectly brittle materials) They are strongly brittle materials such that $\mathrm{w}^{\prime} / E^{\prime}$ is constant. Accordingly, these materials are characterized by the unique state function $\alpha \mapsto E(\alpha)$ which is twice continuously differentiable and must satisfy

$$
E(0)=E_{0}>0, \quad E^{\prime}(\alpha)<0 \quad \forall \alpha \in[0,1), \quad E(1)=0,
$$

while $\mathrm{w}(\alpha)$ is given by

$$
\mathrm{w}(\alpha)=\left(E_{0}-E(\alpha)\right) \frac{\varepsilon_{c}^{2}}{2}
$$

where $\varepsilon_{c}$ is a given positive constant.

2.2 The damage problem of a bar under traction

Let us consider a homogeneous bar whose natural reference configuration is the interval $(0, L)$ and whose crosssectional area is 1 . The bar is made of the nonlocal damaging material characterized by the state function $W$ given by (1). The end $x=0$ of the bar is fixed, while the displacement of the end $x=L$ is prescribed to a value $U_{t}$

$$
u_{t}(0)=0, \quad u_{t}(L)=U_{t} \geq 0, \quad t \geq 0
$$

where, in this quasi-static setting, $t$ denotes the loading parameter or shortly the "time", and $u_{t}$ is the displacement field of the bar at time $t$. The dependence of $U_{t}$ on $t$ is assumed to be smooth, at least continuous and piecewise continuously differentiable. The evolution of displacement and of damage in the bar is obtained using a variational formulation, the main ingredients of which are recalled hereafter, see Benallal and Marigo (2007) for details and Pham and Marigo (2010a,b), Pham et al. (2011a,b) for a general discussion on the variational formulation of damage evolution problems.

For a given $U \in \mathbb{R}$, we denote by $\mathcal{C}_{U}$ the set of "smooth" fields $v$ defined on $[0, L]$ and such that $v(0)=0$, $v(L)=U$, i.e.

$$
\mathcal{C}_{U}=\left\{v \in H^{1}(0, L): v(0)=0, v(L)=U\right\}
$$

where $H^{1}(0, L)$ denotes the usual Sobolev space of functions which belong to $L^{2}(0, L)$ and whose distributional first derivative also belongs to $L^{2}(0, L)$. Accordingly, $\mathcal{C}_{U_{t}}$ and $\mathcal{C}_{\dot{U}_{t}}$ denote the sets of kinematically admissible displacement fields and kinematically admissible displacement rate fields, while $\mathcal{C}_{0}$ is their associated linear space. The set of admissible damage fields is the convex set $\mathcal{D}$ defined by

$$
\mathcal{D}=\left\{\beta \in H^{1}(0, L): 0 \leq \beta(x)<1, \forall x \in[0, L]\right\} .
$$

Let us note that the value 1 for the damage is excluded because some quantities like the compliance $S$ and its derivatives are no more defined when $\alpha=1$. It turns out also that the real displacement field is no more regular but is discontinuous at points $x$ where $\alpha(x)=1$. Since this situation corresponds to the rupture of the bar, we will merely determine at which time $t_{r}$ that happens and the analysis will stop at this moment.

By virtue of the irreversibility condition, damage can only grow and accordingly the convex cone $\dot{\mathcal{D}}$ of admissible damage rate is given by

$$
\dot{\mathcal{D}}=\left\{\beta \in H^{1}(0, L): \beta(x) \geq 0, \forall x \in[0, L]\right\} .
$$

With any admissible pair $(u, \alpha)$, we associate the total energy of the bar

$$
\begin{aligned}
\mathcal{P}(u, \alpha) & :=\int_{0}^{L} W\left(u^{\prime}(x), \alpha(x), \alpha^{\prime}(x)\right) d x \\
& =\int_{0}^{L}\left(\frac{1}{2} E(\alpha(x)) u^{\prime}(x)^{2}+\mathrm{w}(\alpha(x))+\frac{1}{2} E_{0} \ell^{2} \alpha^{\prime}(x)^{2}\right) d x
\end{aligned}
$$

We are in a position to set the evolution problem. Specifically, for a given initial damage field $\alpha^{0}$, the damage evolution problem reads as:

PB 1 (Variational damage evolution problem) Find $t \mapsto\left(u_{t}, \alpha_{t}\right)$ absolutely continuous and such that
1. For all $t \geq 0, \quad\left(u_{t}, \alpha_{t}\right) \in \mathcal{C}_{U_{t}} \times \mathcal{D}$,
2. For almost all $t>0, \quad\left(\dot{u}_{t}, \dot{\alpha}_{t}\right) \in \mathcal{C}_{\dot{U}_{t}} \times \dot{\mathcal{D}}$,
3. For almost all $t>0$ and for all $(v, \beta) \in \mathcal{C}_{\dot{U}_{t}} \times \dot{\mathcal{D}}, \quad \mathcal{P}^{\prime}\left(u_{t}, \alpha_{t}\right)\left(v-\dot{u}_{t}, \beta-\dot{\alpha}_{t}\right) \geq 0$, 
with the initial condition $\alpha_{0}(x)=\alpha^{0}(x)$. In the third item, $\mathcal{P}^{\prime}(u, \alpha)(v, \beta)$ denotes the derivative of $\mathcal{P}$ at $(u, \alpha)$ in the direction $(v, \beta)$ and is given by

$$
\mathcal{P}^{\prime}(u, \alpha)(v, \beta)=\int_{0}^{L}\left(E(\alpha) u^{\prime} v^{\prime}+\left(\frac{1}{2} E^{\prime}(\alpha) u^{\prime 2}+\mathrm{w}^{\prime}(\alpha)\right) \beta+E_{0} \ell^{2} \alpha^{\prime} \beta^{\prime}\right) d x
$$

Remark 1 Note that the second item contains the irreversibility condition $\dot{\alpha}_{t} \geq 0$ and that our formulation makes sense only if the evolution is sufficiently smooth in time. Therefore, we only consider evolutions such that the displacement field and the damage field are absolutely continuous functions of time. To enlarge the search to evolutions which are discontinuous in time, which is often necessary as we will see in the last section, one has to reformulate the evolution problem and replace the third item by a condition which remains meaningful for discontinuous evolutions. This is the essence of the variational formulation proposed in Pham and Marigo (2010a,b) and Pham et al. (2011b) where the third item of PB 1 is replaced by a stability condition and an energy balance. More precisely, the third item is a necessary but in general not sufficient condition in order that the stability condition and the energy balance hold. It merely corresponds to a first order stability condition.

Choosing $\beta=\dot{\alpha}_{t}$ and $v=\dot{u}_{t}+v_{0}$ with $v_{0} \in \mathcal{C}_{0}$ and inserting into the third item of PB 1, we obtain the variational formulation of the equilibrium of the bar,

$$
\int_{0}^{L} E\left(\alpha_{t}(x)\right) u_{t}^{\prime}(x) v_{0}^{\prime}(x) d x=0, \quad \forall v_{0} \in \mathcal{C}_{0}
$$

From (13), we deduce that the stress is constant all along the bar and hence is only a function of time

$$
\sigma_{t}=E\left(\alpha_{t}(x)\right) u_{t}^{\prime}(x), \quad \forall x \in(0, L)
$$

Dividing (14) by $E\left(\alpha_{t}(x)\right)$, integrating over $(0, L)$ and using boundary conditions (8), we find

$$
\sigma_{t} \int_{0}^{L} S\left(\alpha_{t}(x)\right) d x=U_{t}
$$

which gives the overall force-displacement response of the bar once the damage field is known.

To obtain the damage problem which governs the evolution of the damage field in the bar, one inserts (13)-(15) into the third item of PB 1. This leads to the variational inequality governing the evolution of the damage

$$
\int_{0}^{L}\left(2 \mathrm{w}^{\prime}\left(\alpha_{t}\right)-\sigma_{t}^{2} S^{\prime}\left(\alpha_{t}\right)\right)\left(\beta-\dot{\alpha}_{t}\right) d x+\int_{0}^{L} 2 E_{0} \ell^{2} \alpha_{t}^{\prime}\left(\beta^{\prime}-\dot{\alpha}_{t}^{\prime}\right) d x \geq 0
$$

where the inequality must hold for all $\beta \in \dot{\mathcal{D}}$ and almost all $t \geq 0$. Integrating by parts the second integral in (16) leads to a new form of the variational inequality:

$$
\int_{0}^{L}\left(2 \mathrm{w}^{\prime}\left(\alpha_{t}\right)-\sigma_{t}^{2} S^{\prime}\left(\alpha_{t}\right)-2 E_{0} \ell^{2} \alpha_{t}^{\prime \prime}\right)\left(\beta-\dot{\alpha}_{t}\right) d x+2 E_{0} \ell^{2}\left(\alpha_{t}^{\prime}(L)\left(\beta(L)-\dot{\alpha}_{t}(L)\right)-\alpha_{t}^{\prime}(0)\left(\beta(0)-\dot{\alpha}_{t}(0)\right)\right) \geq 0
$$

Setting first $\beta=0$ and then $\beta=2 \dot{\alpha}_{t}$ in (17), we obtain the equality:

$$
\int_{0}^{L}\left(2 \mathrm{w}^{\prime}\left(\alpha_{t}\right)-\sigma_{t}^{2} S^{\prime}\left(\alpha_{t}\right)-2 E_{0} \ell^{2} \alpha_{t}^{\prime \prime}\right) \dot{\alpha}_{t} d x+2 E_{0} \ell^{2}\left(\alpha_{t}^{\prime}(L) \dot{\alpha}_{t}(L)-\alpha_{t}^{\prime}(0) \dot{\alpha}_{t}(0)\right)=0 .
$$

Inserting this equality into (17) leads to the following inequality:

$$
\int_{0}^{L}\left(2 \mathrm{w}^{\prime}\left(\alpha_{t}\right)-\sigma_{t}^{2} S^{\prime}\left(\alpha_{t}\right)-2 E_{0} \ell^{2} \alpha_{t}^{\prime \prime}\right) \beta d x+2 E_{0} \ell^{2}\left(\alpha_{t}^{\prime}(L) \beta(L)-\alpha_{t}^{\prime}(0) \beta(0)\right) \geq 0, \quad \forall \beta \in \dot{\mathcal{D}} .
$$

Choosing first $\beta \in \mathcal{C}_{0}^{\infty}(0, L) \cap \dot{\mathcal{D}}$, where $\mathcal{C}_{0}^{\infty}(0, L)$ denotes the space of indefinitely differentiable functions with compact support in $(0, L)$, the inequality $(18)$ becomes

$$
\int_{0}^{L}\left(2 \mathrm{w}^{\prime}\left(\alpha_{t}\right)-\sigma_{t}^{2} S^{\prime}\left(\alpha_{t}\right)-2 E_{0} \ell^{2} \alpha_{t}^{\prime \prime}\right) \beta d x \geq 0, \quad \forall \beta \in \mathcal{C}_{0}^{\infty}(0, L), \quad \beta \geq 0,
$$

from which we deduce by standard arguments that the following inequality must hold almost everywhere in $(0, L)$ :

$$
2 \mathrm{w}^{\prime}\left(\alpha_{t}\right)-\sigma_{t}^{2} S^{\prime}\left(\alpha_{t}\right)-2 E_{0} \ell^{2} \alpha_{t}^{\prime \prime} \geq 0 .
$$


Choosing now $\beta(x)=(1-x / h)^{+}$in (19) with $0<h<L, a^{+}=\max \{0, a\}$ denoting the positive part of $a$, one gets

$$
\int_{0}^{h}\left(2 \mathrm{w}^{\prime}\left(\alpha_{t}\right)-\sigma_{t}^{2} S^{\prime}\left(\alpha_{t}\right)-2 E_{0} \ell^{2} \alpha_{t}^{\prime \prime}\right)\left(1-\frac{x}{h}\right) d x-2 E_{0} \ell^{2} \alpha_{t}^{\prime}(0) \geq 0 .
$$

Passing to the limit when $h$ goes to 0 , one obtains

$$
\alpha_{t}^{\prime}(0) \leq 0 .
$$

In the same way, choosing $\beta(x)=(1-(L-x) / h)^{+}$one gets

$$
\alpha_{t}^{\prime}(L) \geq 0
$$

Hence, (17) is satisfied only if (20)-(22) are satisfied. Conversely, one immediately sees that if (20)-(22) are satisfied, then (17) is also satisfied. Consequently, (17) and (20)-(22) are equivalent.

Using (20)-(22) and taking into account the irreversibility condition $\dot{\alpha}_{t} \geq 0$, (18) gives the following equalities:

$$
\left(2 \mathrm{w}^{\prime}\left(\alpha_{t}\right)-\sigma_{t}^{2} S^{\prime}\left(\alpha_{t}\right)-2 E_{0} \ell^{2} \alpha_{t}^{\prime \prime}\right) \dot{\alpha}_{t}=0, \quad \alpha_{t}^{\prime}(0) \dot{\alpha}_{t}(0)=0, \quad \alpha_{t}^{\prime}(L) \dot{\alpha}_{t}(L)=0
$$

where the first one must hold almost everywhere in $(0, L)$. Finally, one has obtained the following fundamental local version of the evolution problem PB 1:

PB 2 The pair of absolute continuous functions of time $t \mapsto\left(u_{t}, \alpha_{t}\right) \in \mathcal{C}_{U_{t}} \times \mathcal{D}$ is solution of PB 1 if and only if, for almost all $t \geq 0$, the following conditions hold true

1. Equilibrium : $u_{t}(x)=\sigma_{t} \int_{0}^{x} S\left(\alpha_{t}(y)\right) d y \quad$ and $\quad U_{t}=\sigma_{t} \int_{0}^{L} S\left(\alpha_{t}(y)\right) d y$,

2. Irreversibility : $\dot{\alpha}_{t} \geq 0$ a.e. in $(0, L)$,

3. Damage criterion in the bulk : $2 \mathrm{w}^{\prime}\left(\alpha_{t}\right)-\sigma_{t}^{2} S^{\prime}\left(\alpha_{t}\right)-2 E_{0} \ell^{2} \alpha_{t}^{\prime \prime} \geq 0$ a.e. in $(0, L)$,

4. Consistency condition in the bulk: $\left(2 \mathrm{w}^{\prime}\left(\alpha_{t}\right)-\sigma_{t}^{2} S^{\prime}\left(\alpha_{t}\right)-2 E_{0} \ell^{2} \alpha_{t}^{\prime \prime}\right) \dot{\alpha}_{t}=0$ a.e. in $(0, L)$,

5. Damage boundary condition : $\alpha_{t}^{\prime}(0) \leq 0$ and $\alpha_{t}^{\prime}(L) \geq 0$,

6. Consistency condition at the boundary : $\alpha_{t}^{\prime}(0) \dot{\alpha}_{t}(0)=0$ and $\alpha_{t}^{\prime}(L) \dot{\alpha}_{t}(L)=0$.

Remark 2 We have implicitly assumed that $x \mapsto \alpha_{t}(x)$ is a sufficiently smooth field so that the integration by parts which leads to (17) is licit. The damage criterion in the bulk (20) makes sense provided that $\alpha_{t}$ is at least continuously differentiable. Such a regularity result could be obtained after a careful treatment of the variational inequality (16), but it is outside the scope of the present paper and this regularity property will be admitted.

Remark 3 From the variational approach, we have deduced boundary conditions for the damage field. These natural boundary conditions are due to the fact that no a priori restrictions are imposed to the damage at the boundaries. Of course, these boundary conditions disappear if we assume that the end points of the bar cannot be damaged. In such a case, the sets of admissible damage fields and of admissible rate damage fields become

$$
\mathcal{D}=\left\{\alpha \in H_{0}^{1}(0, L): 0 \leq \alpha<1 \text { in }[0, L]\right\}, \quad \dot{\mathcal{D}}=\left\{\alpha \in H_{0}^{1}(0, L): \alpha \geq 0 \text { in }[0, L]\right\}
$$

and the conditions $\alpha_{t}(0)=\alpha_{t}(L)=0, \dot{\alpha}_{t}(0)=\dot{\alpha}_{t}(L)=0$ replace the items 5 and 6 in the setting of the evolution problem PB 2. More generally, a large variety of boundary conditions can be considered in our variational approach. In any case, by duality, a natural condition is associated with each degree of freedom left by the evolution of damage at the boundary. It is one of the numerous advantages of the variational approach. In the present paper, since no restrictions are imposed to the damage at the boundaries, the homogeneous response is possible whereas the response is necessarily non homogeneous if one constraints the ends to remain undamaged. However, the construction of the localized solution inside the bar does not depend on the damage boundary conditions.

In terms of energy, we have the following property

Property 1 (Balance of energy) Let us assume that the bar is undamaged and unstretched at time 0, i.e. $\alpha_{0}=0$ and $U_{0}=0$. By definition, the work done by the external loads up to time $t$ is given by

$$
\mathcal{W}_{e}(t)=\int_{0}^{t} \sigma_{s} \dot{U}_{s} d s
$$

the total dissipated energy in the bar during the damage process up to time $t$ is given by

$$
\mathcal{E}_{d}(t)=\frac{1}{2} E_{0} \ell^{2} \int_{0}^{L} \alpha_{t}^{\prime}(x)^{2} d x+\int_{0}^{L} \mathrm{w}\left(\alpha_{t}(x)\right) d x
$$


while the elastic energy which remains stored in the bar at time $t$ is equal to

$$
\mathcal{E}_{e}(t)=\frac{\sigma_{t}^{2}}{2} \int_{0}^{L} S\left(\alpha_{t}(x)\right) d x .
$$

By virtue of the conditions of PB 2 that the fields have to satisfy, the following balance of energy holds true at each time:

$$
\mathcal{W}_{e}(t)=\mathcal{E}_{e}(t)+\mathcal{E}_{d}(t)
$$

Proof. By virtue of the equilibrium condition and by definition of the elastic energy, the work done by the external load can read as

$$
\begin{aligned}
\mathcal{W}_{e}(t) & =\int_{0}^{t} \sigma_{s}\left(\dot{\sigma}_{s} \int_{0}^{L} S\left(\alpha_{s}(x)\right) d x+\sigma_{s} \int_{0}^{L} S^{\prime}\left(\alpha_{s}(x)\right) \dot{\alpha}_{s}(x) d x\right) d s \\
& =\int_{0}^{t} \dot{\mathcal{E}}_{e}(s) d s+\int_{0}^{t} \int_{0}^{L} \frac{\sigma_{s}^{2}}{2} S^{\prime}\left(\alpha_{s}(x)\right) \dot{\alpha}_{s}(x) d x d s .
\end{aligned}
$$

Using the initial condition and the consistency condition in the bulk, one gets

$$
\begin{aligned}
\mathcal{W}_{e}(t) & =\mathcal{E}_{e}(t)+\int_{0}^{t} \int_{0}^{L} \mathrm{w}^{\prime}\left(\alpha_{s}\right) \dot{\alpha}_{s} d x d s-E_{0} \ell^{2} \int_{0}^{t} \int_{0}^{L} \alpha_{s}^{\prime \prime} \dot{\alpha}_{s} d x d s \\
& =\mathcal{E}_{e}(t)+\int_{0}^{L} \mathrm{w}\left(\alpha_{t}\right) d x+E_{0} \ell^{2} \int_{0}^{t} \int_{0}^{L} \alpha_{s}^{\prime} \dot{\alpha}_{s}^{\prime} d x d s-E_{0} \ell^{2} \int_{0}^{t}\left(\alpha_{s}^{\prime}(L) \dot{\alpha}_{s}(L)-\alpha_{s}^{\prime}(0) \dot{\alpha}_{s}(0)\right) d s
\end{aligned}
$$

Using once more the initial condition and the consistency condition at the boundary, we obtain the desired equality.

2.3 The homogeneous solution and the issue of uniqueness

If we assume that the bar is undamaged at $t=0$, i.e. if $\alpha^{0}(x)=0$ for all $x$, then it is easy to check that the damage evolution problem admits a solution where $\alpha_{t}$ depends on $t$ but not on $x$. This particular solution will be called the homogeneous solution. Let us construct it in the case where the prescribed displacement is monotonically increasing, i.e. when $U_{t}=t L$ and under the stronger assumption that $\alpha \mapsto-\mathrm{w}^{\prime}(\alpha) / E^{\prime}(\alpha)$ is increasing from a positive value to $+\infty$ when $\alpha$ grows from 0 to 1 (instead of being merely non decreasing as it is stated in Hypothesis 1 , see comment 6 ).

Since we assume spatial homogeneity for $\alpha_{t}$, we have $u_{t}(x)=t x$ and it remains to find the two time functions $t \mapsto \alpha_{t}$ and $t \mapsto \sigma_{t}$. From (15), we get $\sigma_{t}=E\left(\alpha_{t}\right) t$. Inserting this relation into (20) and (23) leads to

$$
\frac{t^{2}}{2} \leq-\frac{\mathrm{w}^{\prime}\left(\alpha_{t}\right)}{E^{\prime}\left(\alpha_{t}\right)}, \quad \dot{\alpha}_{t}\left(\frac{t^{2}}{2}+\frac{\mathrm{w}^{\prime}\left(\alpha_{t}\right)}{E^{\prime}\left(\alpha_{t}\right)}\right)=0 .
$$

Since $\alpha_{0}=0$, the inequality for the damage criterion in (27) is strict at $t=0$ and hence by continuity during a certain time interval. During this time interval, the bar remains undamaged by virtue of the consistency condition in (27). Hence $\alpha_{t}=0$ holds as long as the inequality in (27) remains strict. Therefore $\alpha_{t}$ remains equal to 0 as long as $t \leq \varepsilon_{c}$ with

$$
\varepsilon_{c}:=\sqrt{\frac{2 \mathrm{w}^{\prime}(0)}{-E^{\prime}(0)}}
$$

This corresponds to the elastic phase. For $t>\varepsilon_{c}$, since $-\mathrm{w}^{\prime} / E^{\prime}$ is assumed to be increasing, the first relation of (27) must be an equality. Therefore $\alpha_{t}$ is given by

$$
\alpha_{t}=\left(-\frac{\mathrm{w}^{\prime}}{E^{\prime}}\right)^{-1}\left(\frac{t^{2}}{2}\right)
$$

and grows from 0 to 1 when $t$ grows from $\varepsilon_{c}$ to $\infty$. During this damaging phase, the stress $\sigma_{t}$ is given by

$$
\sigma_{t}=\sqrt{\frac{2 \mathrm{w}^{\prime}\left(\alpha_{t}\right)}{S^{\prime}\left(\alpha_{t}\right)}} .
$$


Since $\mathrm{w}^{\prime} / S^{\prime}$ is decreasing to 0 by virtue of Hypothesis $1, \sigma_{t}$ decreases from $\sigma_{c}$ to 0 when $t$ grows from $\varepsilon_{c}$ to $\infty$ and the critical stress $\sigma_{c}$ is given by

$$
\sigma_{c}:=\sqrt{\frac{2 \mathrm{w}^{\prime}(0)}{S^{\prime}(0)}}=E_{0} \varepsilon_{c}
$$

This last property corresponds to the softening character of the damage model as it was announced in comment 7 after Hypothesis 1 . Note that $\sigma_{t}$ tends only asymptotically to 0 , which means that an infinite displacement is necessary to break the bar in the case of a homogeneous response. The damage rate and the stress rate are discontinuous at $t=\varepsilon_{c}$. Indeed, just before $t_{c}$, one has $\dot{\alpha}_{t}=0$ and $\dot{\sigma}_{t}=E_{0}$, while, just after, one has $\dot{\alpha}_{t}>0$ and $\dot{\sigma}_{t}<0$. For further comparison with non-homogeneous solutions, let us calculate the stress rate at the beginning of the damage phase. Differentiating (29) with respect to $t$ gives the damage rate $\dot{\alpha}_{t}$, while differentiating (30) with respect to $t$ gives the stress rate $\dot{\sigma}_{t}$ in terms of $\dot{\alpha}_{t}$. Combining both relations finally gives

$$
\lim _{t \downarrow \varepsilon_{c}} \dot{\sigma}_{t}=-\frac{E_{0}}{\frac{2 S^{\prime}(0)^{2} \sigma_{c}^{2} E_{0}}{S^{\prime \prime}(0) \sigma_{c}^{2}-2 \mathrm{w}^{\prime \prime}(0)}-1} .
$$

In terms of energy, the dissipated energy during the damage process is given by

$$
\mathcal{E}_{d}(t)=\mathrm{w}\left(\alpha_{t}\right) L
$$

Hence, it is proportional to the length of the bar. The total energy spent to obtain a fully damaged state is equal to $\mathrm{w}(1) L$ and hence is finite by virtue of Hypothesis 1 .

The non local term has no influence on the homogeneous solution. The length of the bar does not play a role and the homogeneous response is the same whatever the bar length.

Let us now examine the issue of the uniqueness of the response. In the case of local damage models (which are obtained by taking $\ell=0$ ), it is well known that the evolution problem admits an infinite number of solution. Does the gradient term ensure the uniqueness? The answer essentially depends on the ratio $L / \ell$ of the bar length with the internal length, as it is proved in Benallal and Marigo (2007) in a particular case and in Pham et al. (2011b) in the general case. Specifically it was shown in (Pham et al. 2011b, Proposition 4.4) that a bifurcation from the homogeneous solution is possible at time $t \geq \varepsilon_{c}$ if and only if $L \geq \mathrm{D}(t)$ with

$$
\mathrm{D}(t)=\pi \ell \sqrt{\frac{2 E_{0}}{\sigma_{t}^{2} S^{\prime \prime}\left(\alpha_{t}\right)-2 \mathrm{w}^{\prime \prime}\left(\alpha_{t}\right)}} .
$$

In particular, a bifurcation can occur at the end of the elastic phase and leads to a non homogeneous damage evolution if $L \geq D_{c}$ with

$$
D_{c}=\pi \ell \sqrt{\frac{2 E_{0}}{\sigma_{c}^{2} S^{\prime \prime}(0)-2 \mathrm{w}^{\prime \prime}(0)}} .
$$

The main goal of the next section is to construct explicitly such a bifurcated solution from the onset of damage to the break of the bar.

Example 2 For the family of models of Example 1, the homogeneous response is given by

$$
\sigma=\left\{\begin{array}{ll}
E_{0} \varepsilon & \text { if } \varepsilon<\varepsilon_{c}=\frac{\sigma_{c}}{E_{0}} \\
\sigma_{c}\left(\frac{\varepsilon}{\varepsilon_{c}}\right)^{\frac{p+q}{p-q}} & \text { if } \varepsilon \geq \varepsilon_{c}
\end{array} \quad \text { and } \quad \alpha=\left\{\begin{array}{ll}
0 & \text { if } \varepsilon<\varepsilon_{c}=\frac{\sigma_{c}}{E_{0}} \\
1-\left(\frac{\varepsilon}{\varepsilon_{c}}\right)^{\frac{2}{p-q}} & \text { if } \varepsilon \geq \varepsilon_{c}
\end{array} .\right.\right.
$$

Since $q>p>0$, the stress is a decreasing function of the strain in the damaging phase. This corresponds to a property of softening. For a given $p>0$, the exponent of the power law goes from $-\infty$ to -1 when $q$ goes from $p$ to $+\infty$. The area under the curve, i.e. the energy dissipated during the full process of homogeneous damage, is finite. This latter feature is typical of strongly brittle behavior, see Pham et al. (2011b).

In the limiting case $p=q$, the damage evolves while the strain remains constant and equal to $\varepsilon_{c}$. This corresponds to a perfectly brittle behavior, see Figure 1 .

In the limiting case $p=0$ or $q=\infty$, the stress-strain curve is an arc of hyperbola in its softening part. The area under the curve, i.e. the energy dissipated during the full process of homogeneous damage, is infinite. This change of the boundedness of the dissipated energy marks the transition between a strongly brittle and a weakly brittle behavior, see Pham et al. (2011b). 

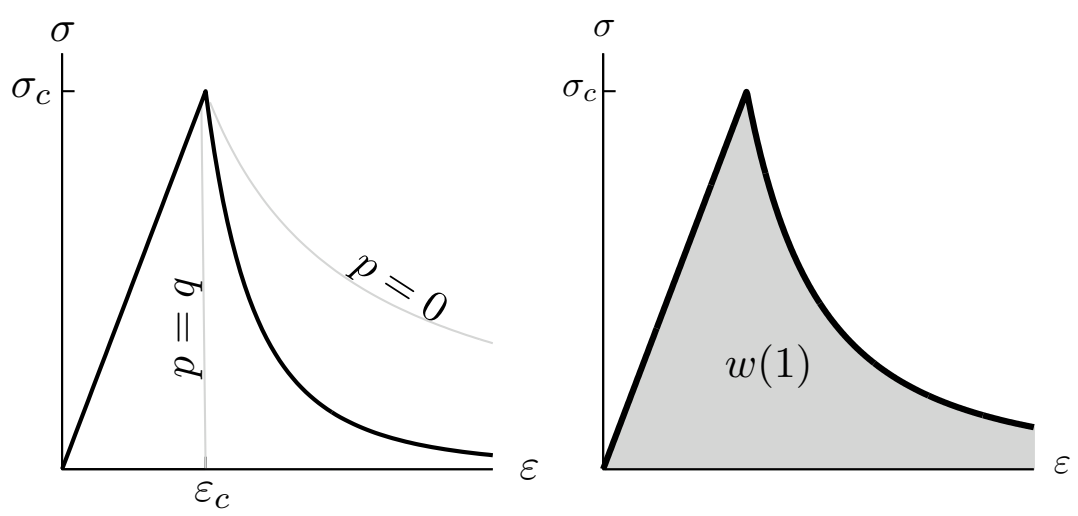

Fig. 1 Left: The stress-strain response (black curve) associated with the homogeneous evolution in the case of the models of Example 1 with $q>p>0$. The limit cases of perfectly brittle material $(p=q)$ and weakly brittle material $(p=0)$ are in gray. Right: Graphical interpretation of the dissipated energy at the end of a homogeneous damage process.

\section{Non homogeneous solutions of the damage problem}

We assume throughout this section that the ratio $L / \ell$ is sufficiently large in order that the boundary conditions at $x=0$ and $x=L$ do not perturb the construction of the non homogeneous solution.

\subsection{The method of construction of non homogeneous solutions}

Let us consider a solution of the evolution problem. We deduce from (20) that $0 \leq \sigma_{t} \leq \sigma_{c}$. Indeed, $\sigma_{t} \geq 0$ by virtue of (8) and (15). Then, integrating (20) over $(0, L)$ and using the boundary conditions (21) and (22), we obtain

$$
\sigma_{t}^{2} \int_{0}^{L} S^{\prime}\left(\alpha_{t}(x)\right) d x \leq \int_{0}^{L} 2 \mathrm{w}^{\prime}\left(\alpha_{t}(x)\right) d x+2 E_{0} \ell^{2}\left(\alpha_{t}^{\prime}(0)-\alpha_{t}^{\prime}(L)\right) \leq \int_{0}^{L} 2 \mathrm{w}^{\prime}\left(\alpha_{t}(x)\right) d x .
$$

But, since $\mathrm{w}^{\prime} / S^{\prime}$ is a decreasing function of $\alpha$ by virtue of Hypothesis 1 and since $\alpha_{t} \geq 0$, we have

$$
2 \mathrm{w}^{\prime}\left(\alpha_{t}(x)\right) \leq \sigma_{c}^{2} S^{\prime}\left(\alpha_{t}(x)\right), \quad \forall x \in(0, L)
$$

Integrating over $(0, L)$ and inserting the result into (34) gives $\sigma_{t}^{2} \leq \sigma_{c}^{2}$. Therefore $\sigma_{c}$ is the maximal stress that the material can sustain in any evolution and not only during a homogeneous damage process.

Let us remark that any solution of the evolution problem contains the same elastic phase, i.e. $\alpha_{t}=0$ as long as $U_{t}$ remains smaller than $\varepsilon_{c} L$. Therefore, damage localizations can appear only when $U_{t}$ has reached the critical value $\varepsilon_{c} L$ and hence $\sigma_{t}$ has reached the critical value $\sigma_{c}$. This critical time is denoted $t_{c}$.

The starting point in the construction of non homogeneous solutions is to seek for solutions for which the equality in (20) holds only in some parts of the bar. For a given $t>t_{c}$, the damage field will be characterized by the set $\mathcal{S}_{t}=\bigcup_{i} \mathcal{S}_{t}^{i}$ made of a finite number of intervals $\mathcal{S}_{t}^{i}$ where $\alpha_{t}>0$. In $[0, L] \backslash \mathcal{S}_{t}$, the material is supposed to be sound and $\alpha_{t}=0$. This part of the bar will be called the (still) elastic part of the bar while the interval $\mathcal{S}_{t}^{i}$ will be called a (damage) localization zone and the damage field inside a (damage) localization profile. We must discriminate an inner localization zone where $\mathcal{S}_{t}^{i}$ is an open interval of the form $\left(x_{i}-D_{t}^{i}, x_{i}+D_{t}^{i}\right) \subset(0, L)$ from a boundary localization zone where $\mathcal{S}_{t}^{i}$ is an interval of the form $\left[0, D_{t}^{i}\right)$ or $\left(L-D_{t}^{i}, L\right]$. To simplify the presentation, we first consider the inner localization zones. We will indicate after what is changed in the case of a boundary localization zone. The successive steps of the construction are as follows:

1. For a given $t>t_{c}$, assuming that $\sigma_{t}$ is known, we determine the profile of the damage field in a localization zone;

2. We study the dependence of the damage profile on the stress $\sigma_{t}$;

3. We analyze under which condition the irreversibility condition is satisfied. 
Let $\sigma_{t}$ be the stress at time $t>t_{c}$, supposed to be known. We know that $\sigma_{t_{c}}=\sigma_{c}$ and that $\sigma_{t}$ cannot be greater than $\sigma_{c}$. The limiting case $\sigma_{t}=\sigma_{c}$ will be treated as a particular case and hence one assumes that $\sigma_{t}<\sigma_{c}$. We will see that $\sigma_{t}=0$ when the damage field takes the critical value 1 somewhere in the bar. This limiting case will also be treated as a particular case. Accordingly, by continuity, we first consider the cases where $\sigma_{t} \in\left(0, \sigma_{c}\right)$.

Throughout the remaining part of this subsection and up to the end of the next one, since $t$ is fixed, we omit the index $t$ in all quantities which are time-dependent. We omit also the index $i$ denoting the size $D$ of the considered localization zone (it will appear that this size is, in fact, the same for all localization zones). Let $\sigma$ be the stress and $\mathcal{S}_{i}=\left(x_{i}-D, x_{i}+D\right)$ be a putative inner localization zone. The damage field $\alpha$ must satisfy

$$
\alpha>0 \quad \text { and } \quad-\sigma^{2} S^{\prime}(\alpha)+2 \mathrm{w}^{\prime}(\alpha)-2 E_{0} \ell^{2} \alpha^{\prime \prime}=0 \quad \text { in } \quad \mathcal{S}_{i} .
$$

Since we assume by construction that the localization zone is matched to an elastic zone where $\alpha=0$ and since $\alpha$ and $\alpha^{\prime}$ must be continuous (see Remark 2), the damage field also has to satisfy the boundary conditions

$$
\alpha\left(x_{i} \pm D\right)=\alpha^{\prime}\left(x_{i} \pm D\right)=0 .
$$

Multiplying (35) by $\alpha^{\prime}$ and integrating with respect to $x$, we obtain the first integral

$$
-\sigma^{2} S(\alpha)+2 \mathrm{w}(\alpha)-E_{0} \ell^{2} \alpha^{\prime 2}=C \text { in } \mathcal{S}_{i},
$$

where $C$ is a constant. Using (36) and Hypothesis 1 , we get $C=-S_{0} \sigma^{2}$ with $S_{0}=1 / E_{0}$ and (37) can read as

$$
\ell^{2} \alpha^{\prime}(x)^{2}=\mathrm{F}(\sigma, \alpha(x)) \text { in } \mathcal{S}_{i}
$$

In $(38), \mathrm{F}$ denotes the function defined in $\left[0, \sigma_{c}\right] \times[0,1)$ by

$$
\mathrm{F}(\sigma, \beta):=2 S_{0} \mathbf{w}(\beta)-S_{0} \sigma^{2}\left(S(\beta)-S_{0}\right)
$$

Since $E_{0} \frac{\partial \mathrm{F}}{\partial \beta}(\sigma, \beta)=2 \mathrm{w}^{\prime}(\beta)-\sigma^{2} S^{\prime}(\beta)$ and since, by virtue of Hypothesis $1, \mathrm{w}^{\prime}(\beta)>0$ and $1-\frac{\sigma^{2} S^{\prime}(\beta)}{2 \mathrm{w}^{\prime}(\beta)}$ decreases from $1-\sigma^{2} / \sigma_{c}^{2}>0$ to $-\infty$ when $\beta$ grows from 0 to 1 , there exists a unique value of $\beta$ in $(0,1)$, say $\alpha^{*}(\sigma)$, for which $\partial \mathrm{F} / \partial \beta$ vanishes:

$$
\alpha^{*}(\sigma)=\left(\frac{\mathrm{w}^{\prime}}{S^{\prime}}\right)^{-1}\left(\frac{\sigma^{2}}{2}\right) .
$$

Accordingly, $\mathrm{F}(\sigma, \cdot)$ vanishes at $\beta=0$, is monotonically increasing in the interval $\left(0, \alpha^{*}(\sigma)\right)$, then is monotonically decreasing in the interval $\left(\alpha^{*}(\sigma), 1\right)$ and tends to $-\infty$ when $\beta$ goes to 1 . Hence there exists a unique value of $\beta$ in $(0,1)$, say $\bar{\alpha}(\sigma)$, for which $\mathrm{F}$ vanishes:

$$
\mathrm{F}(\sigma, \bar{\alpha}(\sigma))=0, \quad \alpha^{*}(\sigma)<\bar{\alpha}(\sigma)<1 .
$$

Since $\alpha>0$ and $\ell \alpha^{\prime}= \pm \sqrt{\mathrm{F}(\sigma, \alpha)}$ in $\mathcal{S}_{i}$, by standard arguments for this type of ordinary differential equations, one deduces that $\alpha^{\prime}\left(x_{i}\right)=0, \alpha\left(x_{i}\right)=\bar{\alpha}(\sigma)$ and

$$
\ell \alpha^{\prime}= \begin{cases}+\sqrt{\mathrm{F}(\sigma, \alpha)} & \text { in }\left(x_{i}-D, x_{i}\right) \\ -\sqrt{\mathrm{F}(\sigma, \alpha)} & \text { in }\left(x_{i}, x_{i}+D\right) .\end{cases}
$$

In other words, $\bar{\alpha}(\sigma)$ corresponds to the maximal value of damage (at the given time), taken at the center of the localization zone. The damage state $\alpha^{*}(\sigma)$ corresponds to the damage state of the bar under the same stress during a homogeneous damage process, see (30). This means that the center part of any localization damage zone is more damaged while the remaining part of the bar is less damaged than in a homogeneous process at the same stress level.

The size of the localization zone is deduced from (41) and (36) by integration. It also depends only on $\sigma$ and is given by

$$
D(\sigma)=\ell \int_{0}^{\bar{\alpha}(\sigma)} \frac{d \beta}{\sqrt{2 S_{0} \mathrm{w}(\beta)-S_{0} \sigma^{2}\left(S(\beta)-S_{0}\right)}} .
$$


Hence $D(\sigma)$ is proportional to the internal length and is finite because the integral is convergent ${ }^{2}$. Provided that $L \geq 2 D(\sigma)$, it is thus possible to insert a localization zone of size $2 D(\sigma)$ inside the bar. The position $x_{i}$ of the center can be chosen arbitrarily in the interval $[D(\sigma), L-D(\sigma)]$.

We finally deduce from (41) and (36) that, in the localization zone, the damage field is given by the following implicit relation between $x$ and $\alpha$ :

$$
\left|x-x_{i}\right|=\ell \int_{\alpha}^{\bar{\alpha}(\sigma)} \frac{d \beta}{\sqrt{2 S_{0} \mathrm{w}(\beta)-S_{0} \sigma^{2}\left(S(\beta)-S_{0}\right)}} .
$$

The damage field is symmetric with respect to the center of the localization zone, decreasing continuously from $\bar{\alpha}(\sigma)$ at the center to 0 at the boundary. The spatial regularity of the damage profile is governed by the regularity of the constitutive functions $\alpha \mapsto \mathrm{w}(\alpha)$ and $\alpha \mapsto S(\alpha)$. Under Hypothesis $1, x \mapsto \alpha(x)$ as a solution of (35) is at least three times continuously differentiable in $\mathcal{S}_{i}$ provided that $\sigma \in\left(0, \sigma_{c}\right)$. The damage profile is even indefinitely differentiable when the constitutive functions are. We will see in the next subsection that this regularity is lost at the limit $\sigma=0$.

Remark 4 The size of an inner localization zone and the damage localization profile depend only on $\sigma$. Since $\sigma$ is a global quantity, all the inner localization zones have the same size and the same profile at a given time. One can also consider localization zones which start at the boundary. In such a case, the consistency condition at the boundary enforces that $\alpha_{t}^{\prime} \dot{\alpha}_{t}$ vanishes at the boundary and consequently the profile is still given by (42)-(43) with $x_{i}=0$ or $x_{i}=L$ and $x \in[0, D(\sigma)]$ or $x \in[L-D(\sigma), L]$. In other words, the profile of a localization zone starting at the boundary is a half of the profile of an inner localization zone, see Figure 2. Accordingly, the total length of the set $\mathcal{S}$ of localization zones is $n D(\sigma)$ with $n$ the number of half-localization zones. Note, however, that such a half-localization zone becomes impossible when one changes the boundary conditions and does not allow that the end of the bar be damaged.

We can summarize our construction of a localized solution by the following property:

Property 2 (Profile of a localized damage field) For a given stress $\sigma \in\left(0, \sigma_{c}\right)$, the damage field in an inner localized damage zone $\left(x_{i}-D(\sigma), x_{i}+D(\sigma)\right)$ is given by (43) while the half-length $D(\sigma)$ of the localized damage zone is finite, proportional to the internal length $\ell$ and given by (42). The damage profile is symmetric with respect to the center $x_{i}$ of the localized damage zone, maximal at the center, the maximal value $\bar{\alpha}(\sigma)$ being given by (40). The damage profile is a continuously differentiable function of $x$, decreasing from $\bar{\alpha}(\sigma)$ at the center to 0 at the boundary of the localized damage zone. The matching with the undamaged part of the bar is smooth, the damage and the gradient of damage vanishing at the boundary of the localized damage zone, see Figure 2.

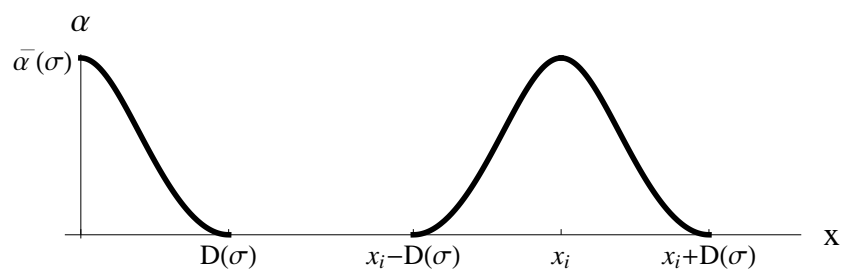

Fig. 2 A typical damage profile in an inner localization zone and in a boundary localization zone when $0<\sigma<\sigma_{c}$

3.3 Dependency of the damage profile on the overall stress

The maximal value of damage depends only on $\sigma$ and enjoys the following property:

Property 3 (Variation of the maximal value of the damage with the stress) When $\sigma$ decreases from $\sigma_{c}$ to 0 , the maximal value $\bar{\alpha}(\sigma)$ taken by the damage field at the center of a localization zone increases from 0 to 1 .

\footnotetext{
2 Indeed, $\mathrm{F}(\sigma, \beta)$ behaves like $\frac{\partial \mathbf{F}}{\partial \beta}(\sigma, 0) \beta$ near $\beta=0$ and like $\frac{\partial \mathbf{F}}{\partial \beta}(\sigma, \bar{\alpha}(\sigma))(\beta-\bar{\alpha}(\sigma))$ near $\beta=\bar{\alpha}(\sigma)$. Since $\frac{\partial \mathbf{F}}{\partial \beta}(\sigma, 0)>0$ and $\frac{\partial \mathrm{F}}{\partial \beta}(\sigma, \bar{\alpha}(\sigma))<0$, the integral is convergent.
} 
Proof. Let $0<\sigma_{1}<\sigma_{2}<\sigma_{c}$. Since $0=\mathrm{F}\left(\sigma_{1}, \bar{\alpha}\left(\sigma_{1}\right)\right)=\mathrm{F}\left(\sigma_{2}, \bar{\alpha}\left(\sigma_{2}\right)\right)<\mathrm{F}\left(\sigma_{1}, \bar{\alpha}\left(\sigma_{2}\right)\right)$, and since $\mathrm{F}\left(\sigma_{1}, \beta\right)<0$ when $\bar{\alpha}\left(\sigma_{1}\right)<\beta<1$, we have $\bar{\alpha}\left(\sigma_{1}\right)>\bar{\alpha}\left(\sigma_{2}\right)$. Hence $\sigma \mapsto \bar{\alpha}(\sigma)$ is decreasing.

Let us prove that $\bar{\alpha}_{0}:=\lim _{\sigma \rightarrow \sigma_{c}} \bar{\alpha}(\sigma)=0$. The limit exists and is non-negative because $\sigma \mapsto \bar{\alpha}(\sigma)$ is monotone and positive on $\left(0, \sigma_{c}\right)$. Passing to the limit in $\mathrm{F}(\sigma, \bar{\alpha}(\sigma))=0$ when $\sigma$ goes to 0 gives $\mathrm{F}\left(\sigma_{c}, \bar{\alpha}_{0}\right)=0$. Since $\mathrm{F}\left(\sigma_{c}, 0\right)=0$ and $\partial \mathrm{F} / \partial \beta\left(\sigma_{c}, \beta\right)<0$ for $\beta>0$, we can conclude that $\bar{\alpha}_{0}=0$.

Let us prove that $\bar{\alpha}_{1}:=\lim _{\sigma \rightarrow 0} \bar{\alpha}(\sigma)=1$. The limit exists and belongs to $(0,1]$ because $\bar{\alpha}(\sigma)$ is decreasing and belongs to $(0,1)$. If $\bar{\alpha}_{1}<1$, passing to the limit in $\mathrm{F}(\sigma, \bar{\alpha}(\sigma))=0$ when $\sigma$ goes to 0 gives $0=\mathrm{F}\left(0, \bar{\alpha}_{1}\right)=2 \mathrm{w}\left(\bar{\alpha}_{1}\right)$, a contradiction. Hence $\bar{\alpha}_{1}=1$.

Let us consider the limiting cases $\sigma=\sigma_{c}$ or $\sigma=0$, i.e. the onset of the damage localization and the moment at which the bar breaks.

Case $\sigma=\sigma_{c}$. In such a case, the differential system (35)-(36) governing the damage profile in a damage localization zone becomes

$$
-\sigma_{c}^{2} S^{\prime}(\alpha)+2 \mathrm{w}^{\prime}(\alpha)-2 E_{0} \ell^{2} \alpha^{\prime \prime}=0 \quad \text { in } \quad \mathcal{S}_{i}, \quad \alpha=\alpha^{\prime}=0 \quad \text { on } \quad \partial \mathcal{S}_{i} .
$$

Integrating the differential equation over $\mathcal{S}_{i}$ and using the boundary conditions lead to

$$
\sigma_{c}^{2} \int_{\mathcal{S}_{i}} S^{\prime}(\alpha) d x=2 \int_{\mathcal{S}_{i}} \mathrm{w}^{\prime}(\alpha) d x
$$

But, by Hypothesis 1 , since $\sigma_{c}^{2} S^{\prime}(\alpha) \leq 2 \mathrm{w}^{\prime}(\alpha)$ for all $\alpha \in[0,1]$ and since the equality holds if and only if $\alpha=0$, the unique solution of the differential equation is $\alpha(x)=0$ for all $x$. This is in agreement with the previous analysis where it was shown that the amplitude of the damage profile tends to 0 when $\sigma$ goes to $\sigma_{c}$. It means that the onset of the damage localization process is progressive as a function of the overall stress. To find the shape of the damage profile when $\sigma$ is close to $\sigma_{c}$, one way is to expand the solution (42)-(43) in terms of the small parameter $\sigma_{c}^{2}-\sigma^{2}$. This requires a careful analysis of the behavior of the integrals when $\sigma$ goes to $\sigma_{c}$. One can show for instance that $\lim _{\sigma \uparrow \sigma_{c}} D(\sigma)=D_{c}>0$ whereas $\lim _{\sigma \uparrow \sigma_{c}} \bar{\alpha}(\sigma)=0$. This means that the onset of damage localization is of small amplitude but occurs in a zone of finite length. This result can be obtained directly by considering the bifurcation equation. Let us follow this latter way as it is customary in bifurcation problems.

Specifically, since we are seeking for small damage fields for $\sigma$ close to $\sigma_{c}$, one linearizes the differential equation (35) which becomes

$$
\alpha>0 \quad \text { and } \quad\left(\sigma_{c}^{2} S^{\prime \prime}(0)-2 \mathrm{w}^{\prime \prime}(0)\right) \alpha+2 E_{0} \ell^{2} \alpha^{\prime \prime}=\left(\sigma_{c}^{2}-\sigma^{2}\right) S^{\prime}(0) \quad \text { in } \quad \mathcal{S}_{i}=\left(x_{i}-D_{c}, x_{i}+D_{c}\right)
$$

the boundary conditions remaining unchanged. To obtain (44) we have taken into account that $S^{\prime}(0) \sigma_{c}^{2}=2 \mathrm{w}^{\prime}(0)$. This is the desired bifurcation equation. Then by standard arguments and after easy calculations which are left to the reader, one gets

$$
\alpha(x)=\frac{2 S^{\prime}(0)\left(\sigma_{c}^{2}-\sigma^{2}\right)}{\sigma_{c}^{2} S^{\prime \prime}(0)-2 \mathrm{w}^{\prime \prime}(0)} \cos ^{2} \frac{\pi\left(x-x_{i}\right)}{2 D_{c}}, \quad D_{c}=\pi \ell \sqrt{\frac{2 E_{0}}{\sigma_{c}^{2} S^{\prime \prime}(0)-2 \mathrm{w}^{\prime \prime}(0)}} .
$$

One has thus obtained the following property:

Property 4 (The onset of a damage localization process) A localization of damage can occur when the stress has reached the critical value $\sigma_{c}$ given by (31). Damage then appears in one (or several) zones of finite size whose half-length $D_{c}$ is given by (45), with a profile which is approximately a sinusoid whose amplitude progressively increases when the stress decreases, see (45).

Note that this value of $D_{c}$ is the same as in (33), which simply means that $D_{c}$ is the minimal length of the bar for which one can construct a non-homogeneous solution at the end of the elastic phase. The localized solution which requires less space is of course the one which starts at one boundary, its size being half of the size of an inner localization zone, see Remark 4.

Example 3 In the case of the family of models of Example 1, the half-length of the damage zone and the amplitude of the damage profile at the onset of damage are given by

$$
\bar{\alpha}(\sigma)=\frac{2}{p+q}\left(1-\frac{\sigma^{2}}{\sigma_{c}^{2}}\right), \quad D_{c}=\sqrt{\frac{2}{(p+q) q}} \frac{\pi \ell}{\varepsilon_{c}} .
$$


Case $\sigma=0$. In this case, our previous construction of the damage profile is not valid. Indeed, the differential system (35)-(36) becomes

$$
\alpha>0 \quad \text { and } \quad \mathrm{w}^{\prime}(\alpha)-E_{0} \ell^{2} \alpha^{\prime \prime}=0 \quad \text { in } \quad \mathcal{S}_{i}, \quad \alpha=\alpha^{\prime}=0 \quad \text { on } \quad \partial \mathcal{S}_{i} .
$$

Integrating the differential equation over $\mathcal{S}_{i}$ and using the boundary conditions leads to $\int_{\mathcal{S}_{i}} \mathrm{w}^{\prime}(\alpha) d x=0$, which is impossible by Hypothesis 1 . As suggested by the fact that the maximal value of damage tends to 1 when $\sigma$ goes to 0 , one has to search for profile such that the damage field takes the value 1 at the center of the zone. Since some quantities like the compliance function $\alpha \mapsto S(\alpha)$ and its derivatives become infinite when $\alpha$ goes to 1 , the regularity of the damage field is lost and $\alpha^{\prime}(x)$ is no more defined at $x=x_{i}$ but undergoes a jump discontinuity. So the differential system now reads

$$
\alpha>0 \quad \text { and } \quad \mathrm{w}^{\prime}(\alpha)-E_{0} \ell^{2} \alpha^{\prime \prime}=0 \quad \text { in } \quad \mathcal{S}_{i} \backslash x_{i}, \quad \alpha\left(x_{i}\right)=1, \quad \alpha=\alpha^{\prime}=0 \quad \text { on } \quad \partial \mathcal{S}_{i} .
$$

Multiplying by $\alpha^{\prime}$ the differential equation valid on each half-zone and taking into account the boundary conditions at the ends, one still obtains a first integral $E_{0} \ell^{2} \alpha^{\prime}(x)^{2}=2 \mathrm{w}(\alpha(x))$ in $\mathcal{S}_{i} \backslash x_{i}$. Since $\alpha>0$ in $\mathcal{S}_{i}$, denoting by $D_{0}$ the half-length of the localization zone, one necessarily has

$$
\ell \alpha^{\prime}= \begin{cases}+\sqrt{2 S_{0} \mathrm{w}(\alpha)} & \text { in }\left(x_{i}-D_{0}, x_{i}\right) \\ -\sqrt{2 S_{0} \mathrm{w}(\alpha)} & \text { in }\left(x_{i}, x_{i}+D_{0}\right) .\end{cases}
$$

Since $\alpha\left(x_{i}\right)=1$, the jump of $\alpha^{\prime}$ at $x_{i}$ is equal to $-2 \sqrt{2 S_{0} \mathrm{w}(1)} / \ell$. By integration, we obtain the damage profile and the half-length of the localization zone:

$$
\left|x-x_{i}\right|=\ell \int_{\alpha}^{1} \frac{d \beta}{\sqrt{2 S_{0} \mathrm{w}(\beta)}}, \quad D_{0}=\ell \int_{0}^{1} \frac{d \alpha}{\sqrt{2 S_{0} \mathrm{w}(\alpha)}} .
$$

One can remark that this solution can be obtained formally by taking $\sigma=0$ and $\bar{\alpha}(0)=1$ in (42)-(43). We have proved the following

Property 5 (Rupture of the bar at the center of a localization zone) At the end of the damage process, when the stress has decreased to 0 , the damage takes the critical value 1 at the center of the localized damage zone. The damage profile and the half length $D_{0}$ of the damage zone are then given by (46). The profile is still symmetric and continuously decreasing to 0 from the center to the boundary, but its slope is discontinuous at the center.

Example 4 In the cases of the family of models of Example 1, the half-length of the damage zone and the amplitude of the damage profile when the bar breaks are given by

$$
\left|x-x_{i}\right|=\frac{\ell}{\varepsilon_{c}} \sqrt{\frac{p}{q}} \int_{0}^{1-\alpha} \frac{d v}{\sqrt{1-v^{p}}}, \quad D_{0}=\frac{\ell}{\varepsilon_{c}} \sqrt{\frac{p}{q}} \int_{0}^{1} \frac{d v}{\sqrt{1-v^{p}}} .
$$

For $p=1$, the profile is made of two symmetric arcs of parabola:

$$
\alpha(x)=\left(1-\frac{\left|x-x_{i}\right|}{D_{0}}\right)^{2}, \quad D_{0}=\frac{2 \ell}{\varepsilon_{c} \sqrt{q}} .
$$

For $p=2$, the profile is made of two symmetric arcs of sinusoid:

$$
\alpha(x)=1-\sin \frac{\pi\left|x-x_{i}\right|}{2 D_{0}}, \quad D_{0}=\frac{\pi \ell}{\varepsilon_{c} \sqrt{2 q}} .
$$

The greater $p$ is, the greater the size of the damage zone and the damage field, see Figure 3.

As we will see in the next subsection, the growth of the localization damage zone when the stress decreases is essential in order to satisfy the irreversibility condition. However, if we compare the size of the damage zone at the onset of damage with its size when the bar breaks, i.e. if we compare $D_{c}$ with $D_{0}$, it is not clear whether $D_{0} \geq D_{c}$. If we consider, for instance, the family of models of Example 1 one has

$$
\frac{D_{0}}{D_{c}}=\frac{\sqrt{p(p+q)} I_{p}}{\pi \sqrt{2}}, \quad I_{p}=\int_{0}^{1} \frac{d v}{\sqrt{1-v^{p}}} .
$$

Since $q \geq p>0$, the inequality $D_{0} \geq D_{c}$ holds only if $p I_{p} \geq \pi$. But, since $p I_{p}$ is an increasing function of $p$ which is equal to $\pi$ when $p=2$, one has $D_{0} \geq D_{c}$ if and only if $p \geq 2$. If we plot the graph of $\sigma \mapsto D(\sigma)$ for different 


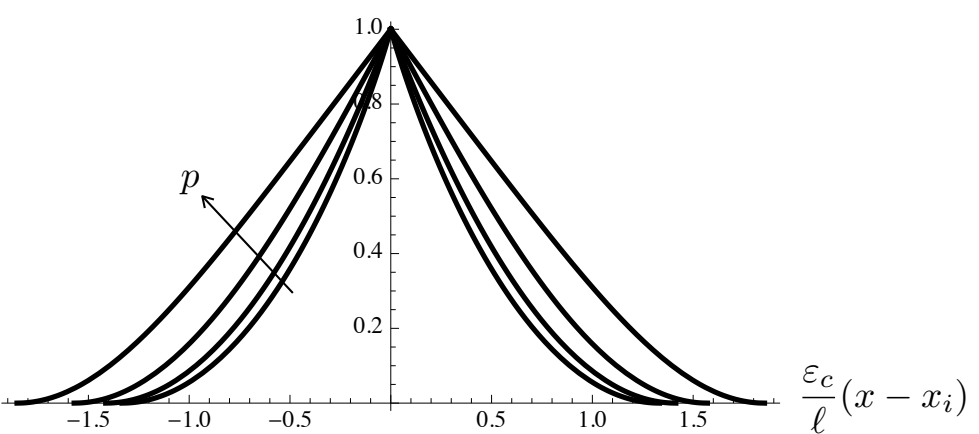

Fig. 3 Damage profile in the localization zone when the bar breaks, for $q=4$ and different values of the parameter $p$ $(p=1 / 2,1,2,4)$ in the family of brittle materials of Example 1

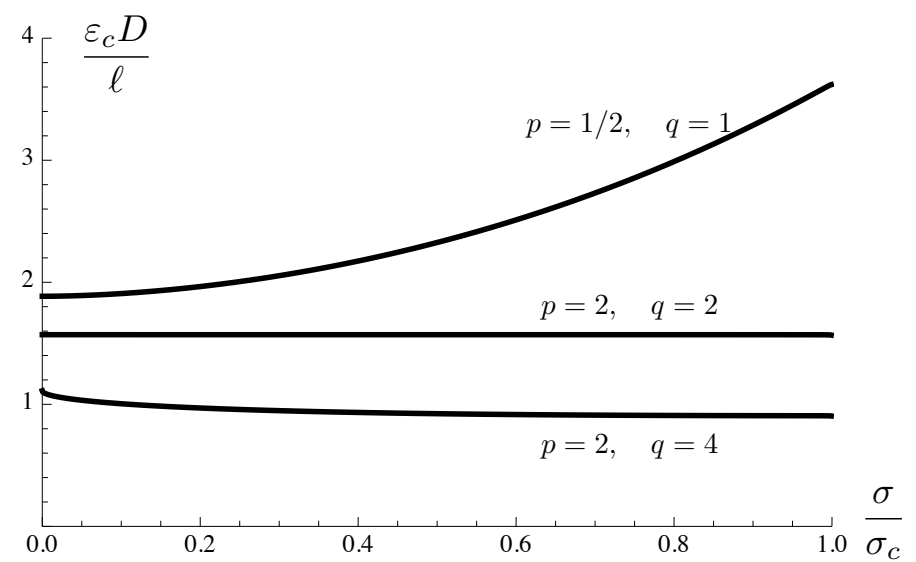

Fig. 4 Evolution of the half-length of the damage zone as a function of the stress for three models of the family of brittle materials of Example 1: for $p=1 / 2$ and $q=2, D$ decreases all along the damage process; for $p=q=2, D$ remains constant; for $p=2$ and $q=4, D$ increases all along the damage process.

values of the parameters, one sees that $D(\sigma)$ is a decreasing function of $\sigma$ only for large enough values of the parameters $p$ and $q$, see Figure 4. So, the monotonicity of $\sigma \mapsto D(\sigma)$ is a material property which depends only on the two state functions $\alpha \mapsto E(\alpha)$ and $\alpha \mapsto \mathrm{w}(\alpha)$. Note that the value of the internal length does not play a role. Since the study of the general case is quite difficult, we will merely establish a sufficient condition for the monotonicity of $\sigma \mapsto D(\sigma)$ in the case of perfectly brittle materials.

Property 6 (Variation of the size of a localization zone with the stress.) For perfectly brittle materials in the sense of Hypothesis 2, if $\alpha \mapsto \sqrt{E(\alpha)}$ is convex, then $\sigma \mapsto D(\sigma)$ is non increasing. In particular, this condition is satisfied in the family of models of Example 1 when $p=q \geq 2$.

Proof. Let us consider a perfectly brittle material and set $\sigma_{c}=E_{0} \varepsilon_{c}, S_{0}=1 / E_{0}$. For $\sigma \in\left(0, \sigma_{c}\right)$, the function $\mathrm{F}$ defined in (39) now reads

$$
\mathrm{F}(\sigma, \alpha)=S_{0}\left(S_{0} \sigma_{c}^{2} E(\alpha)-\sigma^{2}\right) \frac{1-S_{0} E(\alpha)}{E(\alpha)}, \quad \alpha \in[0,1)
$$

The maximal value $\bar{\alpha}(\sigma)$ of the damage is hence given by

$$
\bar{\alpha}(\sigma)=E^{-1}\left(\frac{\sigma^{2}}{\sigma_{c}^{2}} E_{0}\right) .
$$

Inserting into the definition of $D(\sigma)$ yields

$$
D(\sigma)=\ell \int_{0}^{\bar{\alpha}(\sigma)} \sqrt{\frac{E_{0} E(\alpha)}{\left(S_{0} \sigma_{c}^{2} E(\alpha)-\sigma^{2}\right)\left(1-S_{0} E(\alpha)\right)}} d \alpha .
$$


Let us consider the change of variable $\alpha \mapsto \theta$ at given $\sigma$ :

$$
S_{0} E(\alpha)=1-\theta+\theta \frac{\sigma^{2}}{\sigma_{c}^{2}}
$$

$\theta$ increases from 0 to 1 when $\alpha$ increases from 0 to $\bar{\alpha}(\sigma)$. Making this change of variable in the integral giving $D(\sigma)$ yields

$$
D(\sigma)=\frac{\ell}{2 \varepsilon_{c}} \int_{0}^{1} \frac{d \theta}{\left|\Phi^{\prime}(\alpha)\right| \sqrt{\theta(1-\theta)}},
$$

where $\Phi$ stands for the function $\alpha \mapsto \Phi(\alpha):=\sqrt{S_{0} E(\alpha)}$ and $\Phi^{\prime}(\alpha)$ is the derivative of $\Phi$ at $\alpha$. If $\alpha \mapsto \sqrt{E(\alpha)}$ is convex, then $\Phi^{\prime}$ is a non-decreasing function of $\alpha$. Since $E$ is a decreasing function of $\alpha$, so is $\Phi$ and $\left|\Phi^{\prime}(\alpha)\right|=$ $-\Phi^{\prime}(\alpha)$. Hence $\left|\Phi^{\prime}(\alpha)\right|$ is a non increasing function of $\alpha$. Since $\alpha$ is a decreasing function of $\sigma$ at given $\theta \in(0,1)$, $\left|\Phi^{\prime}(\alpha)\right|$ is a non-decreasing function of $\sigma$ at given $\theta$. Accordingly $D$ is a non-increasing function of $\sigma$.

In the case of Example 1, the material is perfectly brittle when $p=q>0$ and then $E(\alpha)=E_{0}(1-\alpha)^{p}$. Hence, $\alpha \mapsto \sqrt{E(\alpha)}$ is convex when $p \geq 2$. Note that when $p=2$, a straightforward calculation gives

$$
D(\sigma)=\frac{\pi \ell}{2 \varepsilon_{c}},
$$

and hence the size of the localization zone remains fixed all along the damage process.

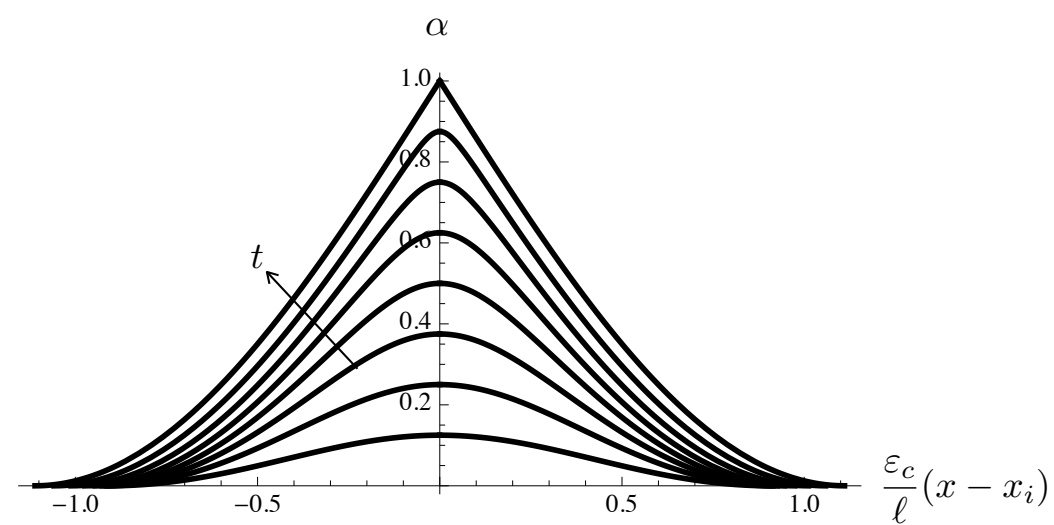

Fig. 5 The damage profile for a given $t$ and its evolution with $t$ by assuming that $t \mapsto \sigma_{t}$ is decreasing in the case of the model of Example 1 with $p=2$ and $q=4$. The rupture occurs when $\sigma_{t}=0$ and $\bar{\alpha}\left(\sigma_{t}\right)=1$. We check numerically that $\sigma \mapsto D(\sigma)$ is decreasing, see Figure 4 .

\subsection{Checking of the irreversibility}

It remains to check that the localized damage fields that we have constructed at different values of $\sigma$ lead to an evolution in time which satisfies the irreversibility condition $\dot{\alpha} \geq 0$. Let us reintroduce the time and the index $t$ in the notation. Since the center of the localization zone is fixed, the condition of irreversibility is satisfied only if $t \mapsto \bar{\alpha}\left(\sigma_{t}\right)=\alpha_{t}\left(x_{i}\right)$ is non-decreasing. Since $\sigma \mapsto \bar{\alpha}(\sigma)$ is decreasing, this is possible only if $t \mapsto \sigma_{t}$ is non-increasing. Since $\alpha_{t}\left(x_{i}, x_{i}+D\left(\sigma_{t}\right)\right)=0$ and since $\alpha_{t}(x)>0$ for $\left|x-x_{i}\right|<D\left(\sigma_{t}\right)$ by construction, the condition of irreversibility is satisfied only if $t \mapsto D\left(\sigma_{t}\right)$ is non decreasing. This requires that $\sigma \mapsto D(\sigma)$ is non increasing, condition which is not automatically satisfied by the damage model, see Figure 4 . When this condition is not satisfied, our construction of localized solutions is no more valid. We must consider an evolution of the damage where a part of the localization zone reenters in a non damaging phase, the size of the still damaging part decreasing with time. To avoid such a situation we make the following hypothesis:

Hypothesis 3 We assume that $\alpha \mapsto E(\alpha)$ and $\alpha \mapsto \mathrm{w}(\alpha)$ are such that $\sigma \mapsto D(\sigma)$ is non increasing.

Note that this hypothesis is satisfied in the class of models of Example 1 when $p=q \geq 2$ by virtue of Property 6 . Under this condition, it is possible to obtain the following property: 
Property 7 Under Hypothesis 3, in order that $t \mapsto \alpha_{t}$ given by (43) in a localization zone (and equals to 0 otherwise) is non-decreasing, it is necessary and sufficient that $t \mapsto \sigma_{t}$ is non-increasing.

Proof. We know that it is necessary, it remains to prove that it is sufficient. Let us assume that $t \mapsto \sigma_{t}$ is non increasing. Then $t \mapsto \bar{\alpha}\left(\sigma_{t}\right)$ and $t \mapsto D\left(\sigma_{t}\right)$ are non decreasing. Let $t_{1}<t_{2}$ and $x$ be such that $\left|x-x_{i}\right| \leq D\left(\sigma_{t_{1}}\right)$. It is sufficient to prove that $\alpha_{2}:=\alpha_{t_{2}}(x) \geq \alpha_{t_{1}}(x)=: \alpha_{1}$. Owing to (43), since $\mathrm{F}$ is a decreasing function of $\sigma$ and since $\sigma_{t_{2}} \leq \sigma_{t_{1}}$, we have

$$
0 \leq D\left(\sigma_{t_{2}}\right)-D\left(\sigma_{t_{1}}\right)=\int_{0}^{\alpha_{2}} \frac{\ell d \beta}{\sqrt{\mathrm{F}\left(\sigma_{t_{2}}, \beta\right)}}-\int_{0}^{\alpha_{1}} \frac{\ell d \beta}{\sqrt{\mathrm{F}\left(\sigma_{t_{1}}, \beta\right)}} \leq \int_{\alpha_{1}}^{\alpha_{2}} \frac{\ell d \beta}{\sqrt{\mathrm{F}\left(\sigma_{t_{1}}, \beta\right)}} .
$$

Hence $\alpha_{2} \geq \alpha_{1}$.

By virtue of this last property, our construction of a non homogeneous solution is valid provided that the bar is sufficiently long for a localization zone to appear and grow without reaching the boundary. Since the size of the localization zone increases with $t$, that leads to the inequality $L \geq 2 D_{0}$. If we consider a non homogeneous solution which starts at one end, our construction is valid provided that the localization zone does not reach the other end of the bar and hence provided that $L \geq D_{0}$. Owing to (46), that gives the following lower bound for $L$ :

$$
L \geq \ell \int_{0}^{1} \frac{d \alpha}{\sqrt{2 S_{0} \mathrm{w}(\alpha)}}
$$

Proposition 1 (A solution of the evolution problem with damage localization) Under Hypotheses 1 and 3, we have constructed a damage evolution $t \mapsto \alpha_{t}$ which satisfies the evolution problem $\mathbf{P B} \mathbf{2}$ if the bar is long enough and if we can control the loading in such a manner that the stress is continuously decreasing. A typical example of the evolution of the damage from its onset to the rupture is given in Figure 5.

In the case where Hypothesis 3 is not satisfied, our construction is no more valid, the irreversibility condition is not satisfied because the size of the damage zone is decreasing. In such a case, it is possible to construct an evolution which satisfies the irreversibility condition by considering the upper envelope of the damage fields, i.e. by considering the evolution $t \mapsto \alpha_{t}^{*}$ such that

$$
\alpha_{t}^{*}(x)=\max _{s \leq t} \alpha_{t}(x), \quad \forall x \in[0, L], \forall t \geq 0
$$

where $\alpha_{t}$ denotes the damage field given by (42)-(43) for a given $\sigma_{t}$. Such a construction is illustrated in Figure 6 for the model corresponding to $p=q=0.5$ in the family of Example 1. For this model, $\sigma \mapsto D(\sigma)$ is decreasing. Note that the difference between the upper envelope and the damage profile initially expected when $\sigma=0$ is important. This means that the true energy dissipated to break the bar is quite greater than the predicted one.

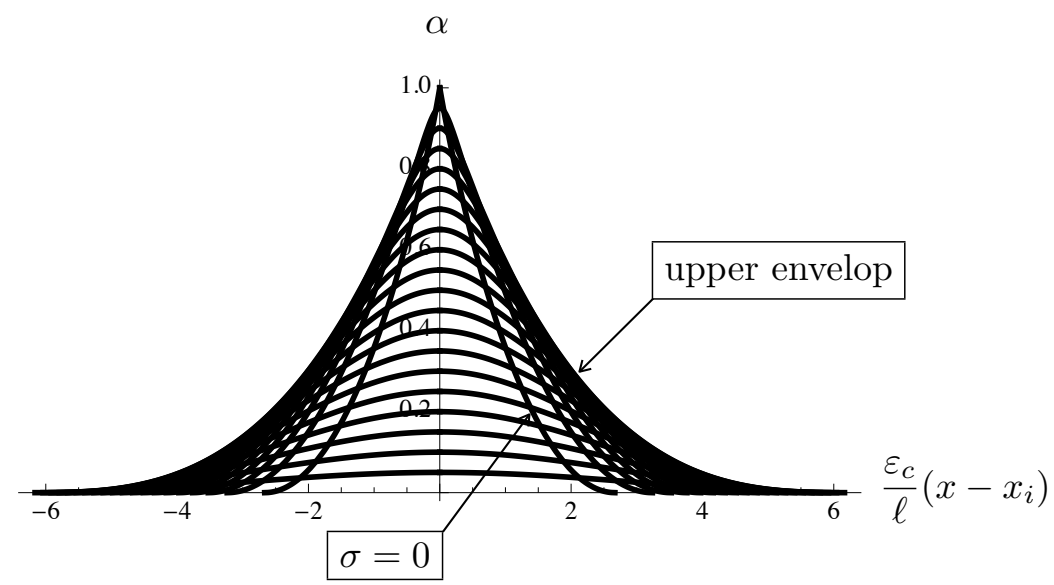

Fig. 6 Construction of an evolution which respects the irreversibility condition in the case where the size of the damage zone predicted by the initial construction decreases while the stress decreases. This consists in taking the upper envelope of the evolution obtained without the irreversibility condition. Here is the case when $p=q=1 / 2$. Note the gap between the upper envelope and the previously considered damage profile when the bar breaks. 


\subsection{Energy dissipated in a localization zone}

By virtue of Property 1 and of (25) the energy dissipated in an inner localization zone when the stress is $\sigma$ is given by

$$
\mathcal{E}_{d}(\sigma)=\int_{x_{i}-D(\sigma)}^{x_{i}+D(\sigma)}\left(\frac{1}{2} E_{0} \ell^{2} \alpha^{\prime}(x)^{2}+\mathrm{w}(\alpha(x))\right) d x .
$$

By symmetry, it is twice the energy dissipated in a half-zone. Using the change of variable $x \rightarrow \alpha$ and (38), we obtain

$$
\mathcal{E}_{d}(\sigma)=\ell \int_{0}^{\bar{\alpha}(\sigma)} \frac{4 \mathrm{w}(\alpha)-\sigma^{2}\left(S(\alpha)-S_{0}\right)}{\sqrt{2 S_{0} \mathrm{w}(\alpha)-S_{0} \sigma^{2}\left(S(\alpha)-S_{0}\right)}} d \alpha .
$$

It is easy to check that $\sigma \mapsto \mathcal{E}_{d}(\sigma)$ is decreasing with $\mathcal{E}_{d}\left(\sigma_{c}\right)=0$, while $\mathcal{E}_{d}(0)$ represents the energy dissipated in a localization zone during the process of damage up to rupture. Let us call fracture energy and denote by $G_{c}$ this energy by reference to the Griffith surface energy density in Griffith's theory of fracture. Since $\bar{\alpha}(0)=1$, we have

Property 8 (Fracture energy) The energy dissipated in an inner localization zone during the damage process up to rupture is a material constant $G_{c}$ which is given by

$$
G_{c}=\ell \int_{0}^{1} \sqrt{8 E_{0} \mathrm{w}(\alpha)} d \alpha .
$$

Because of the lack of constraint on the damage at the boundary, the dissipated energy in a boundary localization zone up to the rupture is $G_{c} / 2$.

Example 5 In the case of the family of strongly brittle materials of Example 1, the fracture energy is given by

$$
G_{c}=2 J_{p} \sqrt{\frac{q}{p}} \sigma_{c} \ell, \quad J_{p}=\int_{0}^{1} \sqrt{1-v^{p}} d v .
$$

Thus $G_{c}$ is proportional to the product of the critical stress by the internal length, the coefficient of proportionality depending on the exponents $p$ and $q$. This link between surface fracture energy, critical stress and internal length is quite similar to the link between the analogous quantities in cohesive force models, see Charlotte et al. (2000); Marigo and Truskinovsky (2004). More generally, cohesive force models and gradient damage models have very similar properties. Both can be seen as regularization of Griffith's model in fracture mechanics, with in each case the great advantage (by comparison to Griffith's model)of containing a critical stress and an internal length. A more fundamental comparison of these two regularized models deserves to be made. The interested reader can refer to Lorentz et al. (2011) for a first interesting attempt in this direction.

\section{The force-displacement relation and global quantities}

In this section we consider the global behavior of the bar during the damage evolution process, in particular the force-displacement response and the different energies involved during the process. We assume that Hypothesis 3 is satisfied and hence that the damage localization profile is given by (43) at a time beyond the elastic phase and when the stress is $\sigma$. The stress is assumed to be controlled during this damaging phase in such a manner that $t \mapsto \sigma_{t}$ is decreasing. How this control can be realized in practice is beyond the scope of this paper. The case when $t \mapsto U_{t}$ is controlled will be considered at the end of the section.

4.1 The additional average strain due to damage

Let $\sigma_{t}$ be the stress prevailing in the bar and $U_{t}$ be the displacement of the end $x=L$ at time $t$. Then, the average strain of the bar is $\varepsilon_{t}=U_{t} / L$. If $t_{c}$ denotes the critical time corresponding to the onset of the damage localization process, one has $\sigma_{t}=E_{0} \varepsilon_{t}$ for $0 \leq t \leq t_{c}$. Let us now consider $t>t_{c}$. Let us assume that the bar contains a time dependent set of localization zones $\mathcal{S}_{t}=\bigcup_{i} \mathcal{S}_{t}^{i}$ whose total length is $n D\left(\sigma_{t}\right)$ with $n \in \mathbb{N}_{*}$ ( $n$ represents the number of half localization zones, recalling that a half-zone can grow at each end). Using (15) and recalling that $\alpha_{t}=0$ outside the damage zone, we get

$$
U_{t}=\sigma_{t} \int_{0}^{L} S\left(\alpha_{t}(x)\right) d x=\sigma_{t}\left(\sum_{i} \int_{\mathcal{S}_{t}^{i}} S\left(\alpha_{t}(x)\right) d x+S_{0}\left(L-n D\left(\sigma_{t}\right)\right)\right) .
$$


The integral $\int_{\mathcal{S}_{t}^{i}} S\left(\alpha_{t}(x)\right) d x$ can be transformed into an integral over the range of $\alpha$ by using (38). Indeed, considering an inner localization zone, by symmetry that integral can read as $2 \int_{x_{i}-D\left(\sigma_{t}\right)}^{x_{i}} S\left(\alpha_{t}(x)\right) d x$. Making the change of variables $x \rightarrow \alpha$, since $\ell d \alpha=\sqrt{\mathrm{F}\left(\sigma_{t}, \alpha\right)} d x$, we obtain

$$
\int_{\mathcal{S}_{t}^{i}} S\left(\alpha_{t}(x)\right) d x=2 \ell \int_{0}^{\bar{\alpha}\left(\sigma_{t}\right)} \frac{S(\alpha) d \alpha}{\sqrt{\mathrm{F}\left(\sigma_{t}, \alpha\right)}} .
$$

Recalling (42), we finally obtain the overall stress-strain relation

$$
\varepsilon_{t}=\varepsilon^{e}\left(\sigma_{t}\right)+\frac{n \ell}{L} \varepsilon^{d}\left(\sigma_{t}\right)
$$

with

$$
\varepsilon^{e}(\sigma)=S_{0} \sigma, \quad \varepsilon^{d}(\sigma)=\int_{0}^{\bar{\alpha}(\sigma)} \frac{\sigma\left(S(\alpha)-S_{0}\right)}{\sqrt{2 S_{0} \mathrm{w}(\alpha)-S_{0} \sigma^{2}\left(S(\alpha)-S_{0}\right)}} d \alpha .
$$

Remark 5 For a given $t$, (51) gives the average strain in term of the stress. When $t$ varies, this corresponds to a curve in the $\varepsilon-\sigma$ plane parametrized by $t$. When $0 \leq t \leq t_{c}$, the response is elastic and one has $\varepsilon=\varepsilon^{e}(\sigma)$ with $0 \leq \sigma \leq \sigma_{c}$. When $t>t_{c}$, since we assume that $t \mapsto \sigma_{t}$ is decreasing, this curve can also be parametrized by $\sigma$ with $\sigma$ decreasing from $\sigma_{c}$ to 0 . Thus $\varepsilon$ can be decomposed into two terms, one associated with a purely elastic response, the other due to the damage of the localization zones. Note that $\sigma \mapsto \varepsilon^{d}(\sigma)$ depends neither on the length of the bar nor on the internal length of the material. The size effects are merely due to the factor $\ell / L$.

The monotonicity properties of the function $\sigma \mapsto \varepsilon^{d}(\sigma)$ depend on the state functions $\alpha \mapsto E(\alpha)$ and $\alpha \mapsto \mathrm{w}(\alpha)$. But, since $\varepsilon^{d}\left(\sigma_{c}\right)=0$ and since $\varepsilon^{d}(\sigma)>0$ for $\sigma<\sigma_{c}, \sigma \mapsto \varepsilon^{d}(\sigma)$ is necessarily decreasing in a neighborhood of $\sigma_{c}$ and we are ensured that $t \mapsto \varepsilon^{d}\left(\sigma_{t}\right)$ is increasing in a neighborhood of $t_{c}$. We have in particular

Property 9 (Behavior of $\sigma \mapsto \varepsilon^{d}(\sigma)$ near $\left.\sigma_{c}\right) \varepsilon^{d}\left(\sigma_{c}\right)=0$ and

$$
\frac{d \varepsilon^{d}}{d \sigma}\left(\sigma_{c}\right)=-\frac{\pi 2^{3 / 2} S^{\prime}(0)^{2} \sigma_{c}^{2} E_{0}^{1 / 2}}{\left(S^{\prime \prime}(0) \sigma_{c}^{2}-2 \mathrm{w}^{\prime \prime}(0)\right)^{3 / 2}} .
$$

Therefore, in the case of the family of models of Example 1 we have

$$
\sigma_{c} \frac{d \varepsilon^{d}}{d \sigma}\left(\sigma_{c}\right)=-\frac{2^{3 / 2} q^{1 / 2} \pi}{(p+q)^{3 / 2}}
$$

Proof. When $\sigma$ is close to $\sigma_{c}$, then $\bar{\alpha}(\sigma)$ is close to 0 and by (45) we have

$$
\bar{\alpha}(\sigma) \approx \frac{2 S^{\prime}(0)\left(\sigma_{c}^{2}-\sigma^{2}\right)}{S^{\prime \prime}(0) \sigma_{c}^{2}-2 \mathrm{w}^{\prime \prime}(0)}
$$

For $\alpha \in(0, \bar{\alpha}(\sigma))$, setting $\alpha=\theta \bar{\alpha}(\sigma)$, the integrand of $\varepsilon^{d}$ is approximately given by

$$
\frac{S(\alpha)-S_{0}}{\sqrt{\mathrm{F}(\sigma, \alpha)}} \approx \frac{\sqrt{2 E_{0}} S^{\prime}(0)}{\sqrt{S^{\prime \prime}(0) \sigma_{c}^{2}-2 \mathrm{w}^{\prime \prime}(0)}} \sqrt{\frac{\theta}{1-\theta}} .
$$

Then, $\varepsilon^{d}(\sigma)$ becomes

$$
\varepsilon^{d}(\sigma) \approx \frac{2^{3 / 2} S^{\prime}(0)^{2} E_{0}^{1 / 2}}{\left(S^{\prime \prime}(0) \sigma_{c}^{2}-2 \mathrm{w}^{\prime \prime}(0)\right)^{3 / 2}} \int_{0}^{1} \sqrt{\frac{\theta}{1-\theta}} d \theta \sigma_{c}\left(\sigma_{c}^{2}-\sigma^{2}\right)
$$

and, since the integral in $\theta$ is equal to $\pi / 2$, the result follows.

On the other hand, the behavior of $\varepsilon^{d}(\sigma)$ when $\sigma / \sigma_{c}$ is small is very sensitive to the constitutive parameters as it is shown in the following example and on Figure 7 (the details of the calculations are left to the reader). 
Example 6 In the case of the family of models of Example 1, we have

$$
\lim _{\sigma \downarrow 0} \varepsilon^{d}(\sigma)= \begin{cases}0 & \text { if } q<2 \\ \pi / 2 & \text { if } q=2 . \\ +\infty & \text { if } q>2\end{cases}
$$

In the case $p=q=2, \varepsilon^{d}(\sigma)$ can be obtained in a closed form in the full range of values of $\sigma$ and one gets

$$
\varepsilon^{d}(\sigma)=\frac{\pi}{2}\left(1-\frac{\sigma}{\sigma_{c}}\right)
$$

Consequently, when $q>2$, the overall strain goes to infinity when the stress goes to 0, contrary to what happens for the homogeneous response in the case $p=q>2$ where the strain remains constant. On the other hand, when $q<2$, the average strain is equal to 0 when the bar breaks. Note however that in some cases, for instance when $p=q<2$, Hypothesis 3 is not satisfied and we should use the upper envelope $\alpha_{t}^{*}$ instead of $\alpha_{t}$.
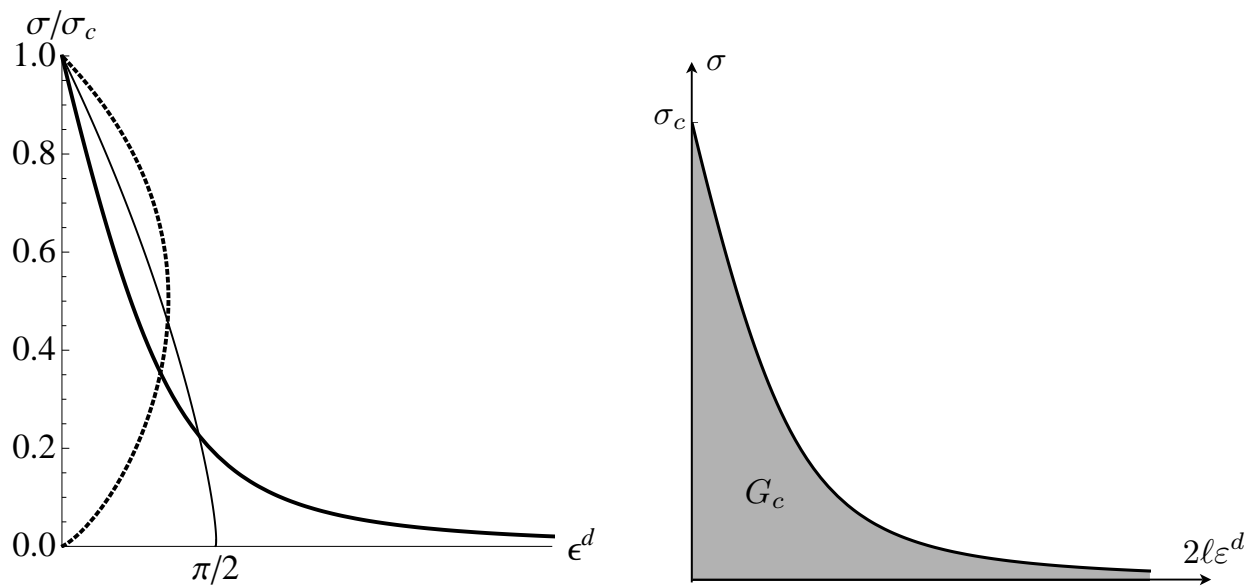

Fig. 7 Left: evolution of $\varepsilon^{d}$ (part of the average strain of the bar due to damage in a localization zone) in a stress-strain diagram. Here are plotted the curves associated with three models of Example 1. Dashed line: $p=0.5, q=1$; thin line: $p=1, q=2$; thick line: $p=2, q=4$. Right : graphical identification of the fracture energy

The value of the fracture energy $G_{c}$ can be seen also in the $\sigma-\varepsilon^{d}$ diagram by virtue of the following

Property 10 (Identification of the fracture energy) The ratio $G_{c} / 2 \ell$ is equal to the area of the region of the $\left(\varepsilon^{d}, \sigma\right)$ plane delimited by the curve $\sigma \mapsto \varepsilon^{d}(\sigma)$ and the $\sigma$ axis, see Figure 7 . Since the product $\ell \varepsilon^{d}(\sigma)$ can be obtained by measuring the displacement $U$ and the stress $\sigma$, this relation can be used to measure the fracture energy.

Proof. Let us calculate this area. Throughout the calculation $\alpha \mapsto \bar{\sigma}(\alpha)$ denotes the inverse function of $\sigma \mapsto \bar{\alpha}(\sigma)$ and we use the fact that $\mathrm{F}(\sigma, \bar{\alpha}(\sigma))=\mathrm{F}(\bar{\sigma}(\alpha), \alpha)=0$ for $\sigma \in\left(0, \sigma_{c}\right)$ and $\alpha \in(0,1)$.

$$
\begin{aligned}
\int_{0}^{\sigma_{c}} \varepsilon^{d}(\sigma) d \sigma & =\int_{0}^{\sigma_{c}} \int_{0}^{\bar{\alpha}(\sigma)} \frac{\sigma\left(S(\alpha)-S_{0}\right)}{\sqrt{2 S_{0} \mathrm{w}(\alpha)-S_{0} \sigma^{2}\left(S(\alpha)-S_{0}\right)}} d \alpha d \sigma \\
& =\int_{0}^{1} \int_{0}^{\bar{\sigma}(\alpha)} \frac{\sigma\left(S(\alpha)-S_{0}\right)}{\sqrt{2 S_{0} \mathrm{w}(\alpha)-S_{0} \sigma^{2}\left(S(\alpha)-S_{0}\right)}} d \sigma d \alpha \\
& =\int_{0}^{1}-\left.\sqrt{2 E_{0} \mathrm{w}(\alpha)-E_{0} \sigma^{2}\left(S(\alpha)-S_{0}\right)}\right|_{\sigma=0} ^{\sigma=\bar{\sigma}(\alpha)} d \alpha \\
& =\int_{0}^{1} \sqrt{2 E_{0} \mathrm{w}(\alpha)} d \alpha
\end{aligned}
$$

Comparing with (49) gives the desired result. 
4.2 Size effects and the force-displacement response

The number $n$ of half-localization zones is limited by the length of the bar. If we consider the onset of damage, we must have $n<L / D_{c}$, while, if we consider the damage process up to the rupture, we must have $n \leq L / D_{0}$. Accordingly, if $L \leq D_{c}$, then no localization can occur and the homogeneous response is the unique solution of the evolution problem.

Let us consider the case $L>D_{c}$. The slope of the stress-strain curve associated with a solution made of $n$ half localization zones, just after $t=t_{c}$, is given by

$$
\frac{d \varepsilon}{d \sigma}\left(\sigma_{c}\right)=S_{0}+\frac{n \ell}{L} \frac{d \varepsilon^{d}}{d \sigma}\left(\sigma_{c}\right):=-S_{0}\left(n \frac{\mathrm{L}_{\mathrm{s}}}{L}-1\right)
$$

where

$$
\mathrm{L}_{\mathrm{s}}:=\ell \frac{\pi 2^{3 / 2} S^{\prime}(0)^{2} \sigma_{c}^{2} E_{0}^{3 / 2}}{\left(S^{\prime \prime}(0) \sigma_{c}^{2}-2 \mathrm{w}^{\prime \prime}(0)\right)^{3 / 2}} .
$$

With the current notation, the slope of the stress-strain curve associated with the homogeneous solution, just after $t=t_{c}$, can read as

$$
\frac{d \varepsilon}{d \sigma}\left(\sigma_{c}\right)=-S_{0}\left(\frac{\mathrm{L}_{\mathrm{s}}}{D_{c}}-1\right)
$$

see (32). Let us remark that the ratio $\mathrm{L}_{\mathrm{s}} / D_{c}$ is not less than 1 since it is given by

$$
\frac{\mathrm{L}_{\mathrm{s}}}{D_{c}}=\frac{2 S^{\prime}(0)^{2} \sigma_{c}^{2} E_{0}}{S^{\prime \prime}(0) \sigma_{c}^{2}-2 \mathrm{w}^{\prime \prime}(0)} .
$$

The inequality $L_{s} \geq D_{c}$ holds by virtue of Hypothesis 1 and of the monotonicity of $-\mathrm{w}^{\prime} / E^{\prime}$. Indeed, since $-2 \mathrm{w}^{\prime}(0) / E^{\prime}(0)=\sigma_{c}^{2} / E_{0}^{2}$ and since $\left(\mathrm{w}^{\prime} / E^{\prime}\right)^{\prime}(0) \leq 0$, a straightforward calculation gives the desired inequality. This inequality ensures that there is no snap-back in the homogeneous response.

If we compare the slope of the homogeneous response with that of a response with $n$ localizations, since $n<L / D_{c}$, one sees that the response with $n$ localizations has a slope at $t=t_{c}$ greater than that of the homogeneous response. This means that the localization induces softening than the homogeneous response, cf Figure 8. Moreover, the response with $n$ localizations will present a snap-back at $t=t_{c}$ if the slope is positive. Accordingly, there is a snap-back when $L>n \mathrm{~L}_{\mathrm{s}}$. We have thus obtained the following property:

Property 11 (Number and shape of the responses with localization) According to the bar length we are in one the three following cases

1. If $L \leq D_{c}$, then no localization zone can appear at $t=t_{c}$ and the homogeneous response is the unique solution;

2. If $D_{c}<L \leq \mathrm{L}_{\mathrm{s}}$, then one or several localization zones can appear inside the bar or at the boundary. The maximal number of half localization zones is $L / D_{c}$. With each possible number of half localization zones is associated a curve in a stress-strain diagram which starts below the curve of the homogeneous response and without snap-back at the bifurcation point;

3. If $L>\mathrm{L}_{\mathrm{s}}$, then the properties are the same as in the previous case except that one or several curves (in particular the curve associated with a unique localization zone located at one end of the bar) present a snack back at the bifurcation point;

The above property is local in time and limited to the behavior of the curves at the onset of damage. Global properties depend both on the model and on the bar length. We give below two examples.

Example 7 In the case of the family of strongly brittle materials of Example 1 with $q>2$ and $q>p>0$, $D_{0}>D_{c}, \lim _{\sigma \rightarrow 0} \varepsilon^{d}(\sigma)=+\infty$ and the two critical lengths are given by

$$
D_{c}=\sqrt{\frac{2}{(p+q) q}} \frac{\pi \ell}{\varepsilon_{c}}, \quad \mathrm{~L}_{\mathrm{s}}=\frac{2 \sqrt{2 q}}{(p+q)^{3 / 2}} \frac{\pi \ell}{\varepsilon_{c}}, \quad \frac{\mathrm{L}_{\mathrm{s}}}{D_{c}}=\frac{2 q}{p+q} .
$$

Since $q>p>0, \mathrm{~L}_{\mathrm{s}} / D_{c} \in(1,2)$. Therefore, one can discriminate the two following cases (cf Figure 8):

1. Case $D_{c}<L \leq \mathrm{L}_{s}<2 D_{c}$. Then the number $n$ of half localization zones is necessarily equal to 1 and there exists a unique bifurcated branch starting at $t=t_{c}$ from the undamaged state. This branch corresponds to a localization zone growing from one end of the bar (by symmetry, the two ends are equivalent). The curve starts below the homogeneous response without snap-back and goes to infinity. 
2. Case $n D_{c} \leq L<(n+1) D_{c}$ with $n \in \mathbb{N}, n \geq 2$. Then there exist $n$ bifurcated branches starting at $t=t_{c}$ from the undamaged state. For $i \in[1, n]$, the $i^{\text {th }}$ branch corresponds to the solutions which contain $i$ half localization zones. When $i$ is odd, such a solution contains necessarily a localization zone located at the boundary, while, when $i$ is even, a solution can contain only inner damage zones as well as inner damage zones with a damage zone at each end of the bar. If $i \mathrm{~L}_{\mathrm{s}}<L$, then the branch presents a snap-back at $t=t_{c}$. In any case the first branch presents a snap-back.
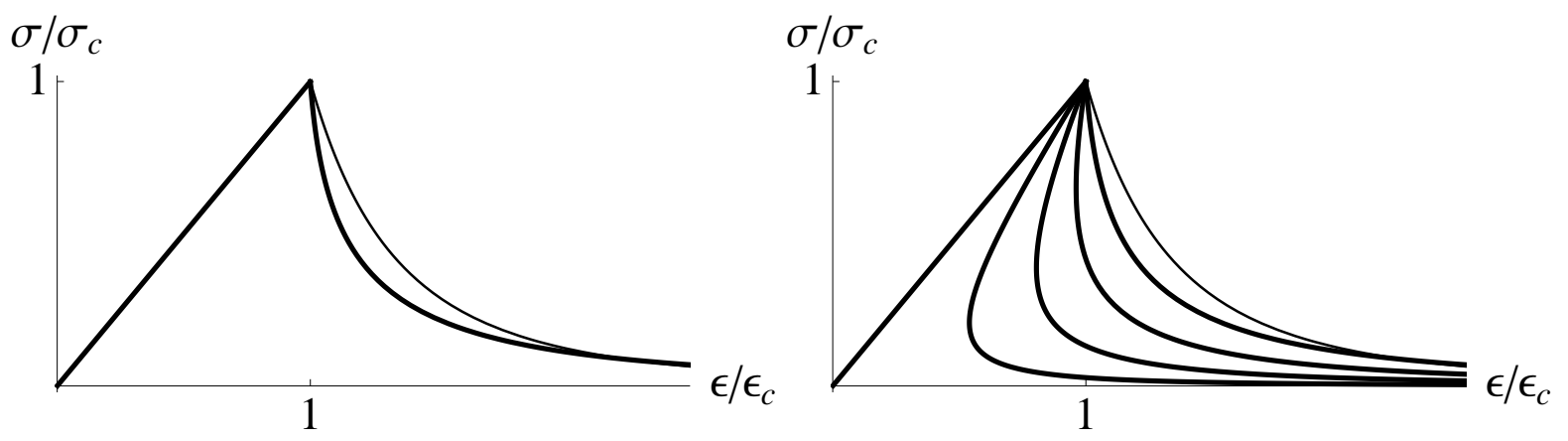

Fig. 8 Overall stress-strain responses for the model of Example 1 with $p=2$ and $q=4$ (hence $\mathrm{L}_{\mathrm{s}}=4 D_{c} / 3$ ). Thin curve: homogeneous response; Thick curves: responses with localization of damage after the elastic phase. Left: for a bar of intermediate length, $D_{c}<L<\mathrm{L}_{s}$, only one boundary localization zone is possible, there is no snap-back; Right: for a long bar, $L>\mathrm{L}_{\mathrm{s}}$ (here $L=4 D_{0} \approx 4.9 D_{c}$ ), several localization zones are possible (here up to 4 ), the first three curves $(1 \leq i \leq 3$ ) present a snap-back, the lowest curve corresponds to $i=1$ and only one half localization zone located at $x=0$ or $x=L$.

Example 8 In the particular case of perfectly brittle materials of Example 1 with $p=q>2$, then $D_{c}=\mathrm{L}_{\mathrm{s}}<$ $D_{0}$ and $\lim _{\sigma \rightarrow 0} \varepsilon^{d}(\sigma)=+\infty$. In the homogeneous response the damage grows at fixed strain. A response with localization is possible only if $L>D_{c}$. In such a case, any curve associated with damage localization presents a snap-back at the damage onset and goes to infinity when the maximal value of the damage goes to 1 (cf Figure 9).

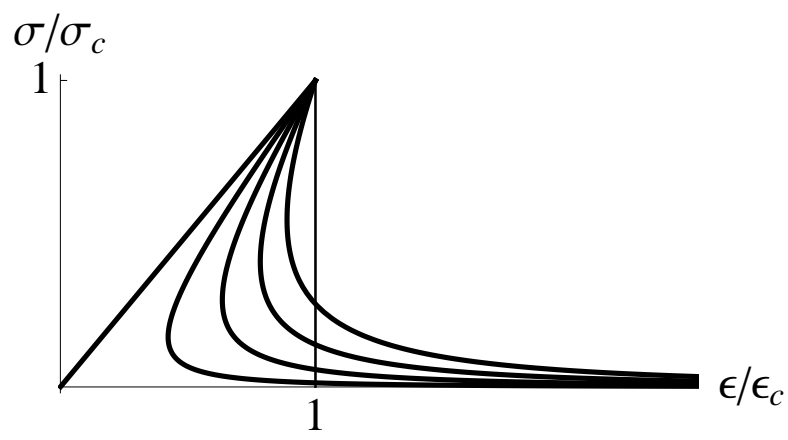

Fig. 9 Overall stress-strain responses for the model of Example 1 with $p=q=4$ (hence $D_{0}=1.67 D_{c}$ ). Thin curve: homogeneous response; Thick curves: responses with localization of damage after the elastic phase. For $L>D_{c}$ (here $L=4 D_{0} \approx 6.67 D_{c}$ ), half localization zones can appear (here up to 6 , but only the first four are plotted), all present a snap-back and go to infinity. The lowest curve corresponds to $i=1$.

4.3 Response of the bar in the case of a hard device control

Let us consider a loading process where $U_{t}=t L$, i.e. such that the displacement of the end $x=L$ is monotonically increasing. The question is: what is the response of the bar just after $t_{c}$ ? We know that the homogeneous response is always possible. But, by Property 11, other possibilities exist provided that the bar length is large enough. In such a case, the question becomes: how to choose among all possible responses? A natural criterion of selection is 
the stability condition proposed in Pham and Marigo (2010b) and used in Pham et al. (2011b) to test the stability of the homogeneous response. We will recall here the main results obtained in Pham et al. (2011b) which allows us to conclude in the neighborhood of the bifurcation point $t=t_{c}$. The analysis of the stability of the states with damage localization in the full range of $t$ is outside the scope of the present paper and is reserved for future works.

The two main results obtained in Pham et al. (2011b) are the following

Property 12 (Instability of the homogeneous state and snap-back on non homogeneous response)

1. If $L<\mathrm{L}_{\mathrm{s}}$, then, at $t=t_{c}$ the undamaged state $\left(\alpha=0, u(x)=t_{c} L\right)$ is stable and there exists an interval of time $\left[t_{c}, t_{c}+h\right)$ such that all the states of a branch solution of the evolution problem which passes by this homogeneous state at time $t_{c}$ are also stable, see (Pham et al. 2011b, Proposition 4.5).

2. If $L>\mathrm{L}_{\mathrm{s}}$, then, at $t=t_{c}$ the undamaged state $\left(\alpha=0, u(x)=t_{c} L\right)$ is unstable, (Pham et al. 2011b, Proposition 3.4).

By virtue of these stability results, let us consider the three cases according to the length of the bar.

1. Case $L \leq D_{c}$. Then, at least in a neighborhood $t=t_{c}$, the homogeneous response is the unique solution and is stable;

2. Case $D_{c}<L \leq \mathrm{L}_{\mathrm{s}}$. Then, at least in a neighborhood $t=t_{c}$, the homogeneous response as well as the possible response with localizations are stable. Accordingly, the damage can grow homogeneously or can appear and grow in a localization zone. The choice can be governed by imperfections or inertial effects;

3. Case $L>L_{s}$. The response is no more homogeneous just after $t_{c}$. But the response associated with the lowest curve (and hence the lowest energy) corresponds to one localization zone at the boundary $(n=1)$ and presents a snap-back. Then, if we assume that the bar prefers the state with the lowest energy, then the response will be discontinuous at time $t_{c}$. Note that if the state of the bar jumps from the undamaged state A to the state of the lowest branch B at the same average strain $t_{c}$, then there is no more conservation of energy, see Figure 10. The additional energy given to the bar during the jump can be transformed either into kinetic energy or into more dissipated energy. In the latter case, the final damage state after the jump will be greater than that corresponding to point B.

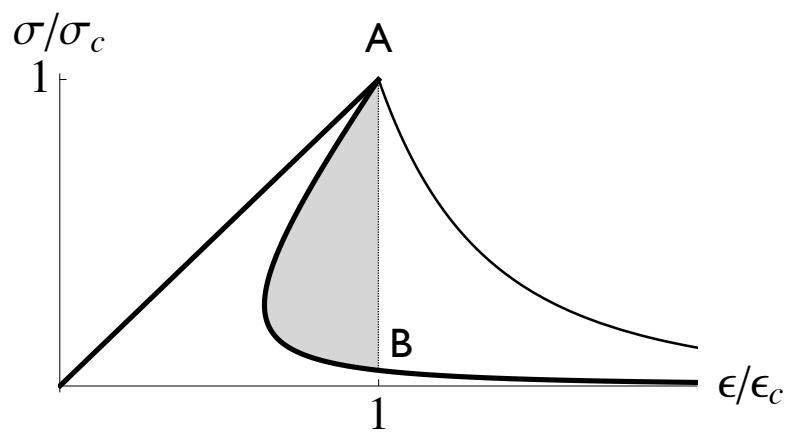

Fig. 10 Case of a long bar $\left(L>L_{s}\right.$. Because of the snap-back of the response with lowest energy and the instability of the homogeneous response (thin curve), the evolution is discontinuous at the bifurcation point $A$. If the state of the bar jumps from $A$ to $B$ then the energy corresponding to the gray area is transformed into kinetic energy or into energy dissipated in further damage.

\section{Conclusion and perspectives}

We have proposed a construction method of non homogeneous solutions for the one-dimensional damage evolution problem of a bar under traction. We have shown that the properties of such localized solutions are very sensitive to the parameters of the model. This strong dependency could be very interesting from an experimental viewpoint to identify the constitutive law and the main parameters of the model as the critical stress, the internal length or the fracture energy.

From a theoretical viewpoint, the presence of the gradient of damage in the model has as expected a regularizing role. It limits the possibility of damage localization since the size of a localization zone is necessarily proportional 
to the internal length of the material, the factor of proportionality depending on the other parameters of the model.

The gradient damage term also induces size effects in the response of the bar. For sufficiently short bars, a damage localization cannot occur and the unique solution is the homogeneous response where the damage field is uniform in the bar. On the other hand, for sufficiently long bars, solutions with several damage localization zones are possible and even more could be constructed, as was made in Benallal and Marigo (2007).

This drastic lack of uniqueness - that the introduction of a non local term has not removed! — requires to add a selection criterion into our formulation of the damage evolution problem. A good candidate is of course the stability criterion introduced in Benallal and Marigo (2007) and Pham and Marigo (2010b). The criterion has been successfully used in Pham et al. (2011b) to study the stability of the homogeneous response and it turned out that the homogeneous damage state is not stable if the bar is long enough. The next challenge is to find which solutions among all those constructed here satisfy the stability criterion. Another important perspective is to extend all these results to a three-dimensional setting.

\section{References}

Bažant, Z. P., T. Belytschko, and T. P. Chang: 1984, 'Continuum theory for strain-softening'. J. Engrg Mech. 110, 16661692.

Bažant, Z. P. and G. Pijaudier-Cabot: 1988, 'Non-local continum damage; localization instability and convergence'. J. Appl. Mech. ASME 55, 287-294.

Benallal, A., R. Billardon, and G. Geymonat: 1993, 'Bifurcation and localization in rate independent materials'. In: Q. Nguyen (ed.): C.S.I.M Lecture Notes on Bifurcation and Stability of Dissipative Systems. Springer-Verlag.

Benallal, A. and J.-J. Marigo: 2007, 'Bifurcation and stability issues in gradient theories with softening'. Modelling Simul. Mater. Sci. Eng. 15, 283-295.

Bourdin, B., G. Francfort, and J.-J. Marigo: 2008, 'The Variational Approach to Fracture'. J. Elasticity 91, 5-148.

Charlotte, M., G. Francfort, J.-J. Marigo, and L. Truskinovsky: 2000, 'Revisiting brittle fracture as an energy minimization problem: Comparisons of Griffith and Barenblatt surface energy models'. In: A. Benallal (ed.): Continuous Damage and Fracture: Proceedings of the symposium, Cachan, France 23-27 octobre 2000. Elsevier.

Comi, C.: 1995, 'On localisation in ductile-brittle materials under compressive loadings'. Eur. J. Mech., A/Solids 14, $19-43$.

Comi, C.: 2001, 'A non-local model with tension and compression damage mechanisms'. European Journal of Mechanics A/Solids 20, 1-22.

Comi, C. and U. Perego: 2001, 'Fracture energy based bi-dissipative damage model for concrete'. International Journal of Solids and Structures 38(36-37), 6427-6454.

de Borst, R., L. Sluys, H.-B. Mühlhaus, and J. Pamin: 1993, 'Fundamental issues in finite element analysis of localization of deformation'. Eng. Comp. 10, 99-121.

DeSimone, A., J.-J. Marigo, and L. Teresi: 2001, 'A damage mechanics approach to stress softening and its application to rubber'. European Journal of Mechanics - A/Solids 20(6), 873-892.

Francfort, G. and J.-J. Marigo: 1993, 'Stable damage evolution in a brittle continuous medium'. Eur. J. Mech. A/Solids 12, 149-189.

Francfort, G. and J.-J. Marigo: 1998, 'Revisiting brittle fracture as an energy minimization'. J. Mech. Phys. Solids 46, $1319-1342$.

Lasry, D. and Belytschko: 1988, 'Localization limiters in transient problems'. Int. J. Solids Structures 24, 581-587.

Lorentz, E. and S. Andrieux: 2003, 'Analysis of non-local models through energetic formulations'. Int. J. Solids Struct. 40, $2905-2936$

Lorentz, E., S. Cuvilliez, and K. Kazymyrenko: 2011, 'Convergence of a gradient damage model toward a cohesive zone model'. Comptes Rendus Mécanique 339(1), 20 - 26.

Marigo, J.-J.: 1989, 'Constitutive relations in Plasticity, Damage and Fracture Mechanics based on a Work Property'. Nuclear Engineering and Design 114, 249-272.

Marigo, J.-J.: 2000, 'From Clausius-Duhem and Drucker-Ilyushin inequalities to standard materials'. In: G. A. Maugin, R. Drouot, and F. Sidoroff (eds.): Continuum Thermodynamics: the art and science of modelling material behaviour, Vol. 76 of Solids Mechanics and Its Applications: Paul Germain's anniversary. Kluwer Acad. Publ.

Marigo, J.-J. and L. Truskinovsky: 2004, 'Initiation and propagation of fracture in the models of Griffith and Barenblatt'. Contin. Mech. Thermodyn. 16(4), 391-409.

Mielke, A.: 2005, 'Evolution of rate-independent systems'. In: Evolutionary equations, Vol. II of Handb. Differ. Equ. Amsterdam: Elsevier/North-Holland, pp. 461-559.

Nguyen, Q.: 1987, 'Bifurcation and postbifurcation analysis in plasticity and brittle fracture'. J. Mech. Phys. Solids 35 , 303-324.

Nguyen, Q. S.: 2000, Stability and Nonlinear Solid Mechanics. London: Wiley \& Son.

Peerlings, R., R. de Borst, W. Brekelmans, J. de Vree, and I. Spee: 1996, 'Some observations on localisation in non-local and gradient damage models'. Eur. J. Mech. A/Solids 15, 937-953.

Peerlings, R., R. de Borst, W. Brekelmans, and M. Geers: 1998, 'Wave propagation and localisation in nonlocal and gradientenhanced damage models'. Journal de Physique IV 8, 293-300.

Pham, K., H. Amor, J.-J. Marigo, and C. Maurini: 2011a, 'Gradient damage models and their use to approximate brittle fracture'. International Journal of Damage Mechanics 20(4), 618-652.

Pham, K. and J.-J. Marigo: 2010a, 'Approche variationnelle de l'endommagement: I. Les concepts fondamentaux'. Comptes Rendus Mécanique 338(4), 191-198. 
Pham, K. and J.-J. Marigo: 2010b, 'Approche variationnelle de l'endommagement: II. Les modèles à gradient'. Comptes Rendus Mécanique 338(4), 199-206.

Pham, K., J.-J. Marigo, and C. Maurini: 2011b, 'The issues of the uniqueness and the stability of the homogeneous response in uniaxial tests with gradient damage models'. J. Mech. Phys. Solids 59(6), 1163-1190.

Pijaudier-Cabot, G. and Z. P. Bažant: 1987, 'Non-local damage theory'. J. Eng. Mech. 113, $1512-1533$.

Pijaudier-Cabot, G. and A. Benallal: 1993, 'Strain localization and bifurcation in a non-local continuum'. Int. J. Solids Struct. 30, 1761-1775. 